The Astrophysical JouRnAL, 554:1175-1196, 2001 June 20

(c) 2001. The American Astronomical Society. All rights reserved. Printed in U.S.A.

\title{
SIMULATIONS OF INCOMPRESSIBLE MAGNETOHYDRODYNAMIC TURBULENCE
}

\author{
Jason MARON ${ }^{1}$ and Peter Goldreich ${ }^{2}$ \\ California Institute of Technology, Pasadena, CA 91125 \\ Received 2000 November 22; accepted 2001 February 9
}

\begin{abstract}
We simulate incompressible MHD turbulence using a pseudospectral code. Our major conclusions are: (1) MHD turbulence is most conveniently described in terms of counterpropagating shear Alfvén and slow waves. Shear Alfvén waves control the cascade dynamics. Slow waves play a passive role and adopt the spectrum set by the shear Alfvén waves. Cascades composed entirely of shear Alfvén waves do not generate a significant measure of slow waves. (2) MHD turbulence is anisotropic, with energy cascading more rapidly along $k_{\perp}$ than along $k_{\|}$, where $k_{\perp}$ and $k_{\|}$refer to wavevector components perpendicular and parallel to the local magnetic field, respectively. Anisotropy increases with increasing $k_{\perp}$ such that excited modes are confined inside a cone bounded by $k_{\|} \propto k_{\perp}^{\gamma}$, where $\gamma<1$. The opening angle of the cone, $\Theta\left(k_{\perp}\right) \propto k_{\perp}^{-(1-\gamma)}$, defines the scale-dependent anisotropy. (3) The one-dimensional inertial range energy spectrum is well fitted by a power law, $E\left(k_{\perp}\right) \propto k_{\perp}^{-\alpha}$, with $\alpha>1$. (4) MHD turbulence is generically strong in the sense that the waves that comprise it suffer order unity distortions on timescales comparable to their periods. Nevertheless, turbulent fluctuations are small deep inside the inertial range. Their energy density is less than that of the background field by a factor of $\Theta^{(\alpha-1) /(1-\gamma)} \ll 1$. (5) MHD cascades are best understood geometrically. Wave packets suffer distortions as they move along magnetic field lines perturbed by counterpropagating waves. Field lines perturbed by unidirectional waves map planes perpendicular to the local field into each other. Shear Alfvén waves are responsible for the mapping's shear and slow waves for its dilatation. The amplitude of the former exceeds that of the latter by $1 / \Theta\left(k_{\perp}\right)$, which accounts for dominance of the shear Alfvén waves in controlling the cascade dynamics. (6) Passive scalars mixed by MHD turbulence adopt the same power spectrum as the velocity and magnetic field perturbations. (7) Decaying MHD turbulence is unstable to an increase of the imbalance between the fluxes of waves propagating in opposite directions along the magnetic field. Forced MHD turbulence displays order unity fluctuations with respect to the balanced state if excited at low $k_{\perp}$ by $\delta(t)$-correlated forcing. It appears to be statistically stable to the unlimited growth of imbalance. (8) Gradients of the dynamic variables are focused into sheets aligned with the magnetic field whose thickness is comparable to the dissipation scale. Sheets formed by oppositely directed waves are uncorrelated. We suspect that these are vortex sheets, which the mean magnetic field prevents from rolling up. (9) Items 1-6 lend support to the model of strong MHD turbulence put forth by Goldreich \& Sridhar (GS). Results from our simulations are also consistent with the GS prediction $\gamma=2 / 3$, as are those obtained previously by Cho \& Vishniac. The sole notable discrepancy is that one-dimensional energy spectra determined from our simulations exhibit $\alpha \approx 3 / 2$, whereas the GS model predicts $\alpha=5 / 3$. Further investigation is needed to resolve this issue.
\end{abstract}

Subject headings: MHD - turbulence

\section{INTRODUCTION}

Most of the baryonic matter in the universe has such high electrical conductivity that magnetic fields diffuse very slowly through it. Thus, fluid motions and motions of magnetic field lines are closely coupled. Large-scale motions are generally turbulent, and incompressible MHD is the simplest approximation under which these complex coupled motions can be investigated.

The inertial range of MHD turbulence is an essential ingredient in a variety of astronomical phenomena. Cosmic rays are scattered by inertial-range magnetic field fluctuations. This affects both their propagation and their acceleration in shock fronts (Blandford \& Eichler 1987; Berezinskii 1990; Chandran 2000). Reconnection of magnetic field lines is an important ingredient of flare activity and dynamo action. The rate at which it proceeds seems likely to depend

\footnotetext{
${ }^{1}$ Current address: Department of Physics, UCLA, 405 Hilgard Avenue, Los Angeles, CA 90095; maron@tapir.caltech.edu.

${ }^{2}$ Caltech MS 150-21; pmg@gps.caltech.edu.
}

on the small-scale structure of magnetic field lines (Parker 1979; Lazarian \& Vishniac 1999). The scintillation of small angular diameter radio sources due to scattering by electron density fluctuations is almost certainly related to inertialrange MHD turbulence (Higdon 1984; Rickett 1990).

The organization of this paper is as follows. Relevant properties of MHD waves are described in $\S 2$. In $\S 3$ we introduce selected analytical models for the inertial range of MHD turbulence. The strategy we follow in designing our simulations is set forth in $\S 4$. Results from these simulations are presented in $\S 5$. In $\S 6$ we interpret our results and compare them with results from prior investigations. Technical details of our simulation method are relegated to the Appendix. Two subsections merit special mention.

A comparison between our simulations and those of earlier workers is given in $\S 6.5$. Here we merely point out that our simulations are most closely related to those presented by Cho \& Vishniac (2000). These authors demonstrate that under nearly isotropic forcing, MHD turbulence develops a scale-dependent anisotropy that increases with increasing wavenumber in the manner suggested by Gold- 
reich \& Sridhar $(1995,1997)$. Henceforth we refer to Goldreich \& Sridhar (1995) and Goldreich \& Sridhar (1997) separately as GSI and GSII, and together as GS. Our simulations differ from those of Cho \& Vishniac (2000) in that they are excited anisotropically, so that we can study the deep inertial range of MHD turbulence.

Our most perplexing result, the shallow slope we find for the one-dimensional energy spectrum, is discussed in $\S$ 6.6. Unfortunately, we cannot offer a definitive explanation. This will require further investigation.

\section{BACKGROUND MATERIAL}

\subsection{Basic Equations}

The equations that govern magnetohydrodynamics (MHD) are written, using notation defined in Table 1,

$$
\begin{gathered}
\rho\left(\partial_{t} v+v \cdot \nabla v\right)=-\nabla\left(p+\frac{B^{2}}{8 \pi}\right)+\frac{1}{4 \pi} \boldsymbol{B} \cdot \nabla \boldsymbol{B}+\rho v_{v} \nabla^{2} v \\
\partial_{t} \boldsymbol{B}=\nabla \times(\boldsymbol{v} \times \boldsymbol{B})+v_{B} \nabla^{2} \boldsymbol{B} \\
\partial_{t} \rho+\nabla \cdot(\rho v)=0 \\
\nabla \cdot \boldsymbol{B}=0
\end{gathered}
$$

The concentration of a passive scalar advected by the fluid evolves according to

$$
\partial_{t} c+\nabla \cdot(c v)=v_{c} \nabla^{2} c .
$$

We simplify equations (1)-(5) for applications in this paper. ${ }^{3}$ Incompressibility is assumed throughout, so we set $\rho=1$ and define the total pressure $P=p+B^{2} / 8 \pi$. The magnetic field is measured in velocity units by $\boldsymbol{b} \equiv \boldsymbol{B} /(4 \pi)^{1 / 2}$. Each diffusive term is replaced by an $n$th order hyperdiffusivity with the same coefficient $v_{n}$. With these modifications, equations (1)-(5) transform to

$$
\begin{gathered}
\partial_{t} v=-\boldsymbol{v} \cdot \nabla \boldsymbol{v}-\nabla P+\boldsymbol{b} \cdot \nabla \boldsymbol{b}+v_{n} \nabla^{2 n} \boldsymbol{v}, \\
\partial_{t} \boldsymbol{b}=-\boldsymbol{v} \cdot \nabla \boldsymbol{b}+\boldsymbol{b} \cdot \nabla \boldsymbol{v}+v_{n} \nabla^{2 n} \boldsymbol{b}, \\
\nabla \cdot \boldsymbol{v}=0, \\
\nabla \cdot \boldsymbol{b}=0, \\
\partial_{t} c+v \cdot \nabla c=v_{n} \nabla^{2 n} c .
\end{gathered}
$$

\footnotetext{
${ }^{3}$ Further steps are taken in $\S$ A.2 to cast these equations in a form suitable for computation.
}

To relate $P$ to $v$ and $b$, we take the divergence of equation (6), which yields

$$
\nabla^{2} P=\nabla b: \nabla b-\nabla v: \nabla v
$$

Thus,

$$
P=\int \frac{d^{3} x^{\prime}}{4 \pi} \frac{(\nabla v: \nabla v-\nabla b: \nabla b)}{\left|x^{\prime}-x\right|}
$$

\subsection{Regimes}

We decompose the magnetic field into a uniform part plus fluctuations,

$$
\boldsymbol{b}=\langle\boldsymbol{b}\rangle+\Delta \boldsymbol{b} .
$$

The Alfvén speed is defined by $v_{\mathrm{A}} \hat{z}=\langle\boldsymbol{b}\rangle ; v_{\mathrm{A}}$ is taken to be constant in space and time, as is consistent with flux conservation. The energy densities of the mean magnetic field, the velocity field, and the magnetic fluctuations are denoted by $E_{\langle\boldsymbol{b}\rangle}, E_{v}$, and $E_{\Delta \boldsymbol{b}}$, respectively. The parameter

$$
\mu=\frac{E_{v}+E_{\Delta \boldsymbol{b}}}{E_{\langle\boldsymbol{b}\rangle}},
$$

which measures the relative importance of the fluctuations compared to the uniform field, determines the character of MHD turbulence.

MHD turbulence with small $\mu$ can be described in terms of interacting waves. Kinetic and potential energy are freely interchanged, so $E_{v}$ and $E_{\Delta b}$ have comparable magnitudes. Wave-mode turbulence is the principal subject of this thesis. Analytic scalings are presented in $\S 3$ to provide an intuitive feel for its dynamics. Results from our simulations are described in $\S 5$ and discussed in $\S 6$.

\subsection{Linear Waves in Incompressible $M H D$}

Linear perturbations about a uniform background magnetic field can be decomposed into shear Alfvén and pseudo Alfvén waves. The pseudo Alfvén wave is the incompressible limit of the slow magnetosonic wave. ${ }^{4}$ As is well known, both waves conform to the dispersion relation

$$
\omega^{2}=v_{\mathrm{A}}^{2} k_{z}^{2} \text {. }
$$

Eigenvectors for these modes take the form

$$
\begin{aligned}
& \hat{\boldsymbol{v}}_{\mathrm{A}}(\boldsymbol{k}, t)=\hat{\boldsymbol{a}}(\boldsymbol{k}) \exp i \boldsymbol{k} \cdot\left(\boldsymbol{x} \mp v_{\mathrm{A}} t \hat{\boldsymbol{z}}\right), \\
& \hat{\boldsymbol{b}}_{\mathrm{A}}(\boldsymbol{k}, t)=\mp \hat{\boldsymbol{a}}(\boldsymbol{k}) \exp i \boldsymbol{k} \cdot\left(\boldsymbol{x} \mp v_{\mathrm{A}} t \hat{\boldsymbol{z}}\right), \\
& \hat{\boldsymbol{v}}_{\mathrm{S}}(\boldsymbol{k}, t)=\hat{\boldsymbol{s}}(\boldsymbol{k}) \exp i \boldsymbol{k} \cdot\left(\boldsymbol{x} \mp v_{\mathrm{A}} t \hat{\boldsymbol{z}}\right), \\
& \hat{\boldsymbol{b}}_{S}(\boldsymbol{k}, t)=\mp \hat{\boldsymbol{s}}(\boldsymbol{k}) \exp i \boldsymbol{k} \cdot\left(\boldsymbol{x} \mp v_{\mathrm{A}} t \hat{z}\right),
\end{aligned}
$$

where the unit polarization vectors are defined by

$$
\hat{\boldsymbol{a}} \equiv \frac{\hat{\boldsymbol{k}} \times \hat{\boldsymbol{z}}}{\left[1-(\hat{\boldsymbol{k}} \cdot \hat{\boldsymbol{z}})^{2}\right]^{1 / 2}}, \quad \hat{\boldsymbol{s}} \equiv \frac{\hat{\boldsymbol{z}}-(\hat{\boldsymbol{k}} \cdot \hat{\boldsymbol{z}}) \hat{\boldsymbol{k}}}{\left[1-(\hat{\boldsymbol{k}} \cdot \hat{\boldsymbol{z}})^{2}\right]^{1 / 2}} .
$$

We note that $\hat{\boldsymbol{k}}, \hat{\boldsymbol{s}}$, and $\hat{\boldsymbol{a}}$ form a right-hand triad.

MHD turbulence is anisotropic, with power cascading more rapidly to high $k_{\perp}$ than to high $k_{z}$. In the limit $k_{\perp} \gg k_{z}$, $\hat{\boldsymbol{s}} \rightarrow \hat{z}$; displacements associated with slow modes align along the unperturbed magnetic field.

\footnotetext{
${ }^{4}$ In the limit of incompressibility, the fast magnetosonic wave has infinite phase velocity and cannot be excited.
} 


\subsection{Elsasser Variables}

The Elsasser transformation

$$
\boldsymbol{w}_{\uparrow}=v_{\mathrm{A}} \hat{z}+\boldsymbol{v}-\boldsymbol{b} \boldsymbol{w}_{\downarrow}=-v_{\mathrm{A}} \hat{z}+\boldsymbol{v}+\boldsymbol{b}
$$

applied to equations (6) and (7) with $v_{n}=0$ brings out the two wave characteristics,

$$
\begin{aligned}
& \partial_{t} \boldsymbol{w}_{\uparrow}+v_{\mathrm{A}} \partial_{z} \boldsymbol{w}_{\uparrow}=-\boldsymbol{w}_{\downarrow} \cdot \nabla \boldsymbol{w}_{\uparrow}-\nabla P, \\
& \partial_{t} \boldsymbol{w}_{\downarrow}-v_{\mathrm{A}} \partial_{z} \boldsymbol{w}_{\downarrow}=-\boldsymbol{w}_{\uparrow} \cdot \nabla \boldsymbol{w}_{\downarrow}-\nabla P,
\end{aligned}
$$

where from equation (12),

$$
P=\int \frac{d^{3} x^{\prime}}{4 \pi} \frac{\nabla \boldsymbol{w}_{\uparrow}: \nabla \boldsymbol{w}_{\downarrow}}{\left|\boldsymbol{x}^{\prime}-\boldsymbol{x}\right|}
$$

Linear waves propagate at the Alfvén speed $v_{\mathrm{A}}$ either parallel $\left(\boldsymbol{w}_{\uparrow}\right)$ or antiparallel $\left(\boldsymbol{w}_{\downarrow}\right)$ to the direction of the background magnetic field.

\subsection{Collisions between Wave Packets}

A disturbance of the background field can be decomposed into upward $\left(\boldsymbol{w}_{\uparrow}\right)$ and downward $\left(\boldsymbol{w}_{\uparrow}\right)$ propagating wave packets. In the special case of unidirectional propagation, either $\boldsymbol{w}_{\uparrow}=0$ or $\boldsymbol{w}_{\downarrow}=0$, and an arbitrary nonlinear wave packet is an exact solution of the equations of incompressible MHD (Parker 1979). To prove this, take the divergence of the equation for the nonzero $w$. This yields $\nabla^{2} P=0$, which, since it applies globally, implies $\nabla P=0$, and hence that the wave packet propagates without distortion. ${ }^{5}$ An important corollary is that nonlinear distortions occur only during collisions between oppositely directed wave packets.

Collisions are constrained by the conservation laws of energy,

$$
E=\frac{1}{2} \int d^{3} x\left(|\boldsymbol{v}|^{2}+|\boldsymbol{b}|^{2}\right),
$$

and cross helicity,

$$
I=\frac{1}{2} \int d^{3} x \boldsymbol{v} \cdot \boldsymbol{b}
$$

These conservation laws follow directly from equations (6)-(9) in the limit that $v_{n}=0$. As a consequence, energy is not exchanged between colliding wave packets. A short proof follows.

Take the dot product of equations (20) and (21) with $\boldsymbol{w}_{\uparrow}$ and $\boldsymbol{w}_{\downarrow}$, respectively. The advective and pressure-gradient terms reduce to total divergences. This establishes that

$$
\frac{d}{d t} \int d^{3} x\left|\boldsymbol{w}_{\uparrow}\right|^{2}=\frac{d}{d t} \int d^{3} x\left|\boldsymbol{w}_{\downarrow}\right|^{2}=0,
$$

provided that $\boldsymbol{w}_{\uparrow}$ and $\boldsymbol{w}_{\downarrow}$ either vanish at infinity or satisfy periodic boundary conditions. From the defining equations for the Elsasser variables, we obtain $\left|\boldsymbol{w}_{\uparrow}\right|^{2} / 2=|\boldsymbol{v}|^{2}+|\boldsymbol{b}|^{2}$ for $\boldsymbol{w}_{\downarrow}=0$ and $\left|\boldsymbol{w}_{\downarrow}\right|^{2} / 2=|\boldsymbol{v}|^{2}+|\boldsymbol{b}|^{2}$ for $\boldsymbol{w}_{\uparrow}=0$. Thus,

$$
E_{\uparrow}=\frac{1}{4} \int d^{3} x\left|\boldsymbol{w}_{\uparrow}\right|^{2} \quad \text { and } \quad E_{\downarrow}=\frac{1}{4} \int d^{3} x\left|\boldsymbol{w}_{\downarrow}\right|^{2}
$$

\footnotetext{
${ }^{5}$ This conclusion remains valid for our simulations, which are carried out in a computational box and employ periodic boundary conditions.
}

are the energies of isolated upward- and downwardpropagating wave packets. This completes the proof that wave packet collisions are elastic.

\subsection{Wave Packets Move along Field Lines}

To lowest nonlinear order in the wave amplitudes, distortions suffered in collisions between oppositely directed wave packets arise because each packet moves along field lines perturbed by the other. The proof follows directly from equation (20), written to second order in the amplitudes of the $\boldsymbol{w}_{\uparrow}$ and $\boldsymbol{w}_{\downarrow}$ fields. With the aid of equation (22), it can be shown that

$$
D_{\uparrow}\left(w_{\uparrow}^{(2)}+\xi^{(1)} \cdot \nabla w_{\uparrow}^{(1)}+\nabla \int \frac{d^{3} x^{\prime}}{4 \pi} \frac{\nabla \xi^{(1)}: \nabla w_{\uparrow}^{(1)}}{\left|x^{\prime}-x\right|}\right)=0 .
$$

Here

$$
D_{\uparrow} \equiv\left(\frac{\partial}{\partial t}+v_{\mathrm{A}} \frac{\partial}{\partial z}\right)
$$

and

$$
\boldsymbol{x}\left(\boldsymbol{x}_{0}, t\right) \equiv \boldsymbol{x}_{0}+\boldsymbol{\xi}\left(\boldsymbol{x}_{0}, t\right) .
$$

The Lagrangian displacement, $\xi$, connects the Lagrangian coordinate of a fluid particle, $\boldsymbol{x}_{0}$, to its Eulerian coordinate, $\boldsymbol{x}$.

Several steps are needed to establish equation (27). In terms of $\boldsymbol{\xi}$,

$$
\boldsymbol{v}=\left.\frac{\partial \boldsymbol{\xi}}{\partial t}\right|_{x_{0}}, \quad \boldsymbol{b}=v_{\mathrm{A}} \hat{z}+\left.v_{\mathrm{A}} \frac{\partial \boldsymbol{\xi}}{\partial z_{0}}\right|_{t} .
$$

To first order in the amplitudes of $\boldsymbol{w}_{\uparrow}$ and $\boldsymbol{w}_{\downarrow}$, we can replace $x_{0}$ by $\boldsymbol{x}$ in the definition of $\boldsymbol{\xi}$ and write

$$
\boldsymbol{v}^{(1)}=\left.\frac{\partial \boldsymbol{\xi}^{(1)}}{\partial t}\right|_{\boldsymbol{x}}, \quad \boldsymbol{b}^{(1)}=\left.v_{\mathrm{A}} \frac{\partial \boldsymbol{\xi}^{(1)}}{\partial z}\right|_{t} .
$$

It then follows from equations (19) and (31) that

$$
\boldsymbol{w}_{\downarrow}^{(1)}=D_{\uparrow} \boldsymbol{\xi}^{(1)} \text {. }
$$

The final step is to verify that the linear operator $D_{\uparrow}$ passes through the integral sign and changes $\boldsymbol{\xi}^{(1)}$ to $\boldsymbol{w}_{\downarrow}^{(1)}$ while leaving the rest of the integrand unaltered.

Equation (27) has a simple interpretation. Consider an upward-moving wave packet for which $\boldsymbol{w}_{\uparrow}^{(2)}=0$ prior to its interaction with downward-moving waves. Subsequent to this interaction, suppose that $\xi^{(1)}$ at fixed $z_{\uparrow}=z-v_{\mathrm{A}} t$ is changed by $\Delta \xi^{(1)}$. Then, as a function of $z_{\uparrow}$,

$$
\Delta w_{\uparrow}^{(2)}=-\Delta \xi^{(1)} \cdot \nabla w_{\uparrow}^{(1)}-\nabla \int \frac{d^{3} x^{\prime}}{4 \pi} \frac{\nabla \Delta \xi^{(1)}: \nabla w_{\uparrow}^{(1)}}{\left|x^{\prime}-x\right|} .
$$

The first term on the right-hand side of this equation is the perturbation that would result from the unconstrained displacement of $\boldsymbol{w}_{\uparrow}^{(1)}$ by $\Delta \boldsymbol{\xi}^{(1)}$. The second term constrains the perturbation to preserve $\boldsymbol{\nabla} \cdot \boldsymbol{w}_{\uparrow}^{(2)}=0 .{ }^{6}$ Since magnetic field lines are frozen in the fluid, we conclude that, at least to second order, wave packets follow magnetic field lines.

Two points are worth stressing in connection with equation (33):

1. Downward-propagating waves contribute the entire $\Delta \xi^{(1)}$, since it is measured at fixed $z_{\uparrow}=z-v_{\mathrm{A}} t$. This is

\footnotetext{
${ }^{6}$ This term arises from the gradient of the total pressure.
} 
consistent with the general rule that only oppositely directed wave packets interact.

2. The turbulent energy cascade is associated with the shear of the $\Delta \xi^{(1)}$ field. Uniform displacements, which arise as a consequence of the sweeping of small disturbances by larger ones, do not contribute to the transfer of energy across scales.

The proof in this section has been couched in Eulerian coordinates. A technically simpler version in Lagrangian coordinates is given by Sridhar \& Goldreich (1994). It consists of demonstrating that the third-order Lagrangian density for incompressible MHD vanishes when written in terms of the transverse components of the displacement vector. Although simpler technically, the Lagrangian-based result is more subtle conceptually. Its proper interpretation is provided in GSII.

\section{MHD CASCADES}

A variety of models have been proposed for MHD turbulence. They share the common feature that energy cascades from lower to higher wavenumber.

\subsection{The Iroshnikov-Kraichnan Model}

The standard model is that of Iroshnikov (1963) and Kraichnan (1965). Kraichnan's derivation of the IK (Iroshnikov-Kraichnan) spectrum relies on the fact that only oppositely directed waves interact in incompressible MHD. It assumes explicitly that the turbulence is isotropic and implicitly that the dominant interactions are those that couple three waves.

The above assumptions imply that the cascade time across scale $\lambda$ is

$$
t_{c} \sim\left(\frac{v_{\mathrm{A}}}{v_{\lambda}}\right)^{2} \frac{\lambda}{v_{\mathrm{A}}} .
$$

Setting $v_{\lambda}^{2} / t_{c}$ equal to the dissipation rate per unit mass, $\epsilon$, then yields

$$
v_{\lambda} \sim\left(\epsilon v_{\mathrm{A}} \lambda\right)^{1 / 4},
$$

which corresponds to the one-dimensional power spectrum, ${ }^{7}$

$$
E(k) \sim \frac{\left(\epsilon v_{\mathrm{A}}\right)^{1 / 2}}{k^{3 / 2}} .
$$

Nonlinearity is measured by $\chi \sim\left(v_{\lambda} / v_{\mathrm{A}}\right)$, where $N \sim \chi^{-2}$ is the number of wave periods in $t_{c}$;

$$
\chi \sim\left(\frac{\epsilon \lambda}{v_{\mathrm{A}}^{3}}\right)^{1 / 4} .
$$

Since $\chi$ decreases with decreasing $\lambda$, only dissipation limits the length of the IK inertial range.

The IK model is flawed because the assumption of isotropy is inconsistent with the frequency and wavevector closure relations that resonant triads must satisfy (Shebalin, Matthaeus, \& Montgomery 1983). These take the form

$$
\begin{gathered}
\omega_{1}+\omega_{2}=\omega_{3}, \\
\boldsymbol{k}_{1}+\boldsymbol{k}_{2}=\boldsymbol{k}_{3} .
\end{gathered}
$$

\footnotetext{
${ }^{7}$ Because the IK cascade is isotropic, it is sufficient to specify its onedimensional power spectrum.
}

However, since $\omega=v_{\mathrm{A}}\left|k_{z}\right|$, equation (38) and the $z$ component of equation (39) yield the set

$$
\begin{gathered}
\left|k_{1 z}\right|+\left|k_{2 z}\right|=\left|k_{3 z}\right|, \\
k_{1 z}+k_{2 z}=k_{3 z} .
\end{gathered}
$$

Because nonlinear interactions can only occur between oppositely directed waves, the three-mode coupling coefficient vanishes unless waves 1 and 2 propagate in opposite directions. In that case, equations (40) and (41) imply that either $k_{1 z}$ or $k_{2 z}$ must vanish. Since one of the incoming waves has zero frequency, three-wave interactions do not cascade energy along $k_{z}$.

\subsection{Intermediate MHD Turbulence}

GSII propose an anisotropic MHD cascade based on scalings obtained from three-wave interactions. It represents a new form of turbulence, which they term intermediate, because it shares some of the properties of both weak and strong turbulence. Although individual wave packets suffer small distortions in single collisions, interactions of all orders make comparable contributions to the perpendicular cascade. $^{8}$

To derive the scaling relations for the intermediate cascade, we repeat the steps carried out in $\S 3.1$ for the IK model, but with $\lambda_{\perp}$ in place of $\lambda$ and $\lambda_{\|}$held constant. Here $\lambda_{\perp}$ and $\lambda_{\|}$are correlation lengths in directions perpendicular and parallel to the local magnetic field, respectively. Thus,

$$
t_{c} \sim\left(\frac{v_{\mathrm{A}} \lambda_{\perp}}{v_{\lambda_{\perp}} \lambda_{\|}}\right)^{2} \frac{\lambda_{\|}}{v_{\mathrm{A}}} .
$$

Setting $\epsilon \sim v_{\lambda_{1}}^{2} / t_{c}$, we find

$$
v_{\lambda_{\perp}} \sim\left(\frac{\epsilon v_{\mathrm{A}} \lambda_{\perp}^{2}}{\lambda_{\|}}\right)^{1 / 4},
$$

and

$$
E\left(k_{\perp}\right) \sim \frac{\left(\epsilon v_{\mathrm{A}} k_{\|}\right)^{1 / 2}}{k_{\perp}^{2}} .
$$

In addition to being anisotropic, the intermediate MHD cascade differs from the IK cascade in another important respect. The strength of nonlinear interactions, as measured by

$$
\chi \sim\left(\frac{v_{\lambda_{\perp}} \lambda_{\|}}{v_{\mathrm{A}} \lambda_{\perp}}\right) \sim\left(\frac{\epsilon \lambda_{\|}^{3}}{v_{\mathrm{A}}^{3} \lambda_{\perp}^{2}}\right)^{1 / 4},
$$

increases along the cascade. Thus, even in the absence of dissipation, the intermediate cascade has a finite inertial range. This suggests that a strong form of MHD turbulence must be the relevant one for most applications in nature.

\subsection{Strong MHD Turbulence}

A cascade for strong MHD turbulence is described in GSI. Its defining property is that MHD waves suffer order unity distortions on timescales comparable to their periods. This state is referred to as one of critical balance. Motivation for the hypothesis of critical balance is given in Goldreich \& Sridhar $(1995,1997)$ and summarized below. Our discussion of intermediate turbulence shows that $\chi$ increases

\footnotetext{
${ }^{8}$ This is a controversial claim.
} 
if it is less than unity. However, it cannot rise above unity, since the frequency spread of the wave packets that emerge following a strong collision must satisfy the frequency-time uncertainty relationship. A. Gruzinov (2000 private communication) provides a more physical explanation for the upper bound on $\chi$. He points out that for $\chi \gg 1$, twodimensional motions of scale $\lambda_{\perp}$ in planes perpendicular to the local magnetic field are uncoupled over separations greater than $\lambda_{\|} / \chi$ along the field direction. Thus, during a time interval of the order of $\lambda_{\perp} / v_{\lambda_{\perp}} \sim \lambda_{\|} / v_{\mathrm{A}} \chi$, these motions reduce $\chi$ to order unity.

Critical balance, and the assumption of a constant energy flux along the cascade, as expressed by

$$
\epsilon \sim \frac{v_{\lambda_{\perp}}^{3}}{\lambda_{\perp}}
$$

imply

$$
\lambda_{\|} \propto \lambda_{\perp}^{2 / 3} .
$$

Although there is a parallel cascade of energy in strong MHD turbulence, the degree of anisotropy increases along the cascade.

Let us assume $v_{L} \sim v_{\mathrm{A}}$ and isotropy on outer scale $L$. Then the three-dimensional energy spectrum of strong MHD turbulence takes the form

$$
E\left(k_{\perp}, k_{\|}\right) \sim \frac{v_{\mathrm{A}}^{2}}{L^{1 / 3} k_{\perp}^{10 / 3}} f\left(\frac{k_{\|} L^{1 / 3}}{k_{\perp}^{2 / 3}}\right),
$$

where $f(u)$ is a positive symmetric function of $u$ with the properties that $f(u) \approx 1$ for $|u| \lesssim 1$ and $f(u)$ is negligibly small for $|u| \gg 1$. The power spectrum is flat as a function of $k_{\|}$for $k_{\|} \lesssim k_{\perp}^{2 / 3} L^{-1 / 3}$ because the velocity and magnetic perturbations on transverse scale $k_{\perp}^{-1}$ arise from independent wave packets whose lengths $\lambda_{\|} \sim \lambda_{\perp}^{2 / 3} L^{1 / 3}$. The onedimensional perpendicular power spectrum obtained from equation (48) reads

$$
E\left(k_{\perp}\right) \sim \frac{v_{\mathrm{A}}^{2}}{L^{2 / 3} k_{\perp}^{5 / 3}} .
$$

Thus, the spectrum of strong MHD turbulence is an anisotropic version of the Kolmogorov (1941) spectrum for hydrodynamic turbulence.

Inertial-range velocity differences and magnetic perturbations across the perpendicular scale $\lambda_{\perp}$ satisfy

$$
v_{\lambda_{\perp}} \sim b_{\lambda_{\perp}} \sim\left(\frac{\lambda_{\perp}}{L}\right)^{1 / 3} v_{\mathrm{A}} .
$$

Thus, even though the turbulence is properly classified as strong, deep in the inertial range magnetic field lines are nearly parallel across perpendicular separations $\lambda_{\perp}$ and nearly straight along parallel separations $\lambda_{\|}$; differential bending angles are of the order of $\left(\lambda_{\perp} / L\right)^{1 / 3} \sim\left(\lambda_{\|} / L\right)^{1 / 2}$.

\subsubsection{Parallel Cascade}

It is interesting to examine the frequency-changing interactions that drive the parallel cascade. Referring back to the intermediate cascade, we know that three-wave interactions do not change frequencies. However, interactions involving more than three waves can. For example, frequency changes arise in four-wave interactions of the form

$$
\omega_{1}+\omega_{2}+\omega_{3}=\omega_{4},
$$

$$
k_{1}+k_{2}+k_{3}=k_{4} \text {, }
$$

where $k_{1 z}$ and $k_{2 z}$ have the same sign and $\omega_{3}=v_{\mathrm{A}}\left|k_{3 z}\right|=0$ (Ng \& Bhattacharjee 1996; GKII). The parallel cascade they give rise to proceeds at a rate that is smaller than that of the perpendicular cascade by a factor of the order of $\chi$. Because strong MHD turbulence is characterized by $\chi \sim 1$, it has a significant parallel cascade.

\subsubsection{Field Line Geometry}

MHD turbulence is best understood geometrically. Field lines perturbed by waves propagating in one direction define two-dimensional mappings between $x y$ planes separated by distance $z$. Shear Alfvén waves dominate the shear and slow waves the dilatation of these mappings. The magnitude of the shear exceeds that of the dilatation by a factor of the order of $\lambda_{\|} / \lambda_{\perp} \sim\left(L / \lambda_{\perp}\right)^{1 / 3} \gg 1$. These mappings describe the distortion that counterpropagating waves would suffer if they moved at uniform speed along the perturbed field lines. The dominance of the shear over the dilatation explains why shear Alfvén waves control the perpendicular cascades of both types of wave.

The recognition that MHD waves tend to follow field lines is essential to understanding their turbulent cascades. Figure 1 provides a visual illustration of how this works. The left panel displays a snapshot of field lines perturbed by downward-propagating waves. In the right panel we follow the evolution of a horizontal pattern as it propagates from the bottom to the top following these lines. The distortion of the initially circular bulls-eye is principally due to the shear in the two-dimensional mapping defined by the perturbed field lines. The cascade time on the scale of the initial pattern is that over which the shear grows to order unity.

This geometrical picture requires two qualifications. The first is that the propagation speed of MHD waves is not exactly constant but varies with the strength of the local magnetic field. Pressure perturbations associated with slow waves are balanced by perturbations of magnetic pressure. The resulting perturbations in propagation speed, of the order of $v_{\lambda_{\perp}}$, contribute to the nonlinear cascade. Over one wave period they lead to fractional distortions of the order of $v_{\lambda_{\perp}} / v_{\mathrm{A}} \sim \lambda_{\perp} / \lambda_{\|} \ll 1$. Thus they are properly ignored. The second qualification is that MHD waves do not exactly follow field lines. The extent to which this affects their cascade remains to be quantified.

The parallel cascade can also be viewed in geometrical terms. Consider an upward-propagating wave packet of length $\lambda_{\|}$and width $\lambda_{\perp}$ that is being distorted by downward-moving wave packets of similar scale. Correlations along the parallel direction are shortened because the front and back of the wave packet undergo different two-dimensional mappings. This happens because the upward-propagating packet distorts each downward-going packet as it passes through it. This distortion is of order $\chi$. For strong MHD turbulence $\chi \sim 1$, which accounts for its significant parallel cascade.

Incidentally, the geometrical picture also aids the interpretation of results from perturbation theory. For example, the three-wave resonant interactions that dominate the perpendicular cascade and the four-wave resonant interactions that cause the lowest order frequency changes each depend on the amplitudes of modes with $k_{z}=0$. This is because the shear in the mapping between $x y$ planes separated by $\Delta z$ is proportional to the displacement amplitudes of modes with 

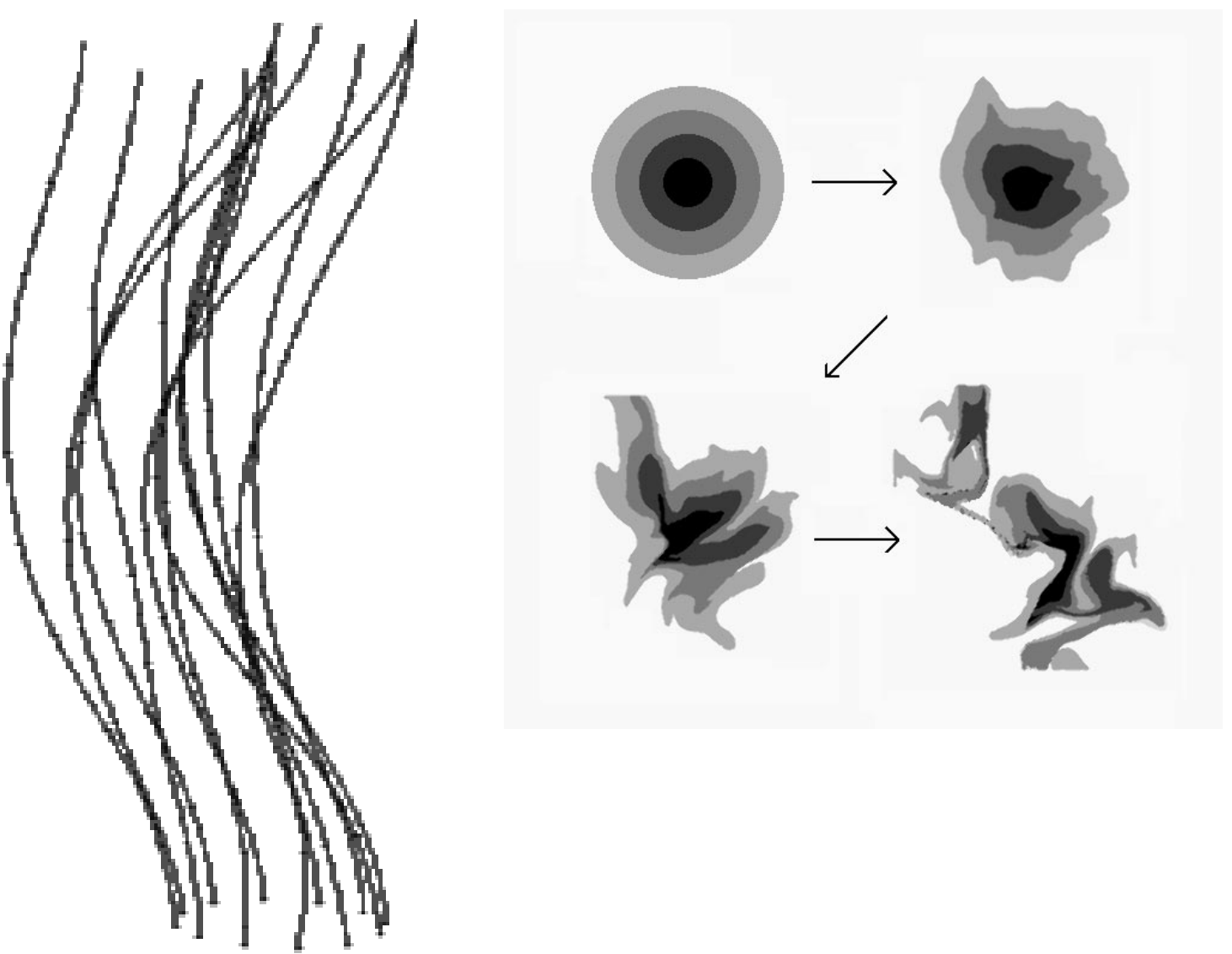

FIG. 1.-Wave packet distortion through field-line wander. Left: Sample of field lines perturbed by downward-propagating waves. Right: Distortion of an originally circular bulls-eye pattern as it moves upward following these field lines.

$k_{z} \lesssim 1 / \Delta z$. Perturbation theory corresponds to the limit of vanishing cascade strength in which shears of order unity are achieved in the limit of infinite separation along the $z$-axis.

\subsubsection{Relation to Two-Dimensional Hydrodynamic Turbulence}

Magnetic field lines possess a tension that makes them ill-disposed to bend, but they are easily shuffled. This accounts for the two-dimensional character of MHD turbulence. It also prompts an inquiry into the relation of MHD turbulence to two-dimensional hydrodynamic turbulence. Each fluid element conserves its vorticity in inviscid twodimensional hydrodynamics. This results in a direct cascade of enstrophy (vorticity squared) toward high $k_{\perp}$ and an inverse cascade of energy toward small $k_{\perp}$ (Lesieur 1990). As we now demonstrate, MHD turbulence does not share these characteristics.

The vorticity equation in MHD, obtained by taking the curl of equation (6) with $v_{n}=0$, reads

$$
\frac{\partial(\boldsymbol{\nabla} \times \boldsymbol{v})}{\partial t}=\boldsymbol{\nabla} \times[\boldsymbol{v} \times(\boldsymbol{\nabla} \times \boldsymbol{v})-\boldsymbol{b} \times(\boldsymbol{\nabla} \times \boldsymbol{b})] .
$$

We concentrate on shear Alfvén waves, since they dominate the field-aligned vorticity for nearly perpendicular cascades. Scaling the terms in equation (53) shows that

$$
\frac{\partial \omega_{\|}}{\partial t} \sim \frac{v_{\lambda_{\perp}}}{\lambda_{\perp}} \omega_{\|} .
$$

Thus, $\omega_{\|}$changes on the cascade timescale; it is not even approximately conserved. Consequently, there is no ens- trophy constraint to prevent energy from cascading toward larger $k_{\perp}$.

\section{SIMULATION STRATEGY}

What follows is a comprehensive discussion of the techniques used in our simulations. Technical aspects of the spectral method are presented in the Appendix.

\subsection{Spectral Wave Mode Decomposition}

Separation of $\tilde{\boldsymbol{v}}(\boldsymbol{k})$ and $\tilde{\boldsymbol{b}}(\boldsymbol{k})$ into upward- and downwardpropagating components is accomplished by forming Fourier coefficients of the Elsasser variables $\tilde{\boldsymbol{w}}_{\uparrow}(\boldsymbol{k})$ and $\tilde{\boldsymbol{w}}_{\downarrow}(\boldsymbol{k})$ according to

$$
\tilde{\boldsymbol{w}}_{\uparrow}(\boldsymbol{k})=\tilde{\boldsymbol{v}}(\boldsymbol{k})-\tilde{\boldsymbol{b}}(\boldsymbol{k}), \quad \tilde{\boldsymbol{w}}_{\downarrow}(\boldsymbol{k})=\tilde{\boldsymbol{v}}(\boldsymbol{k})+\tilde{\boldsymbol{b}}(\boldsymbol{k}) .
$$

Projections of $\tilde{\boldsymbol{w}}_{\uparrow}(\boldsymbol{k})$ and $\tilde{\boldsymbol{w}}_{\downarrow}(\boldsymbol{k})$ along the polarization directions of the linear incompressible MHD eigenmodes given by equation (18) yield amplitudes of upward- and downward-propagating Alfvén and slow waves. In obvious notation,

$$
\begin{aligned}
A_{\uparrow}(\boldsymbol{k}) & \equiv \hat{\boldsymbol{a}} \cdot \tilde{\boldsymbol{w}}_{\uparrow}(\boldsymbol{k})=\tilde{v}_{\mathrm{A}}(\boldsymbol{k})-\tilde{b}_{\mathrm{A}}(\boldsymbol{k}), \\
S_{\uparrow}(\boldsymbol{k}) & \equiv \hat{\boldsymbol{s}} \cdot \tilde{\boldsymbol{w}}_{\uparrow}(\boldsymbol{k})=\tilde{v}_{S}(\boldsymbol{k})-\tilde{b}_{S}(\boldsymbol{k}), \\
A_{\downarrow}(\boldsymbol{k}) & \equiv \hat{\boldsymbol{a}} \cdot \tilde{\boldsymbol{w}}_{\downarrow}(\boldsymbol{k})=\tilde{\boldsymbol{v}}_{\mathrm{A}}(\boldsymbol{k})+\tilde{b}_{\mathrm{A}}(\boldsymbol{k}), \\
S_{\downarrow}(\boldsymbol{k}) & \equiv \hat{\boldsymbol{s}} \cdot \tilde{\boldsymbol{w}}_{\downarrow}(\boldsymbol{k})=\tilde{v}_{S}(\boldsymbol{k})+\tilde{b}_{\mathrm{S}}(\boldsymbol{k}),
\end{aligned}
$$

where

$$
\begin{array}{cc}
\tilde{v}_{\mathrm{A}}(\boldsymbol{k})=\hat{\boldsymbol{a}} \cdot \tilde{\boldsymbol{v}}(\boldsymbol{k}), & \tilde{b}_{\mathrm{A}}(\boldsymbol{k})=\hat{\boldsymbol{a}} \cdot \tilde{\boldsymbol{b}}(\boldsymbol{k}), \\
\tilde{v}_{S}(\boldsymbol{k})=\hat{\boldsymbol{s}} \cdot \tilde{\boldsymbol{v}}(\boldsymbol{k}), & \tilde{b}_{S}(\boldsymbol{k})=\hat{\boldsymbol{s}} \cdot \tilde{\boldsymbol{b}}(\boldsymbol{k}) .
\end{array}
$$


The eigenmode frame, $(\hat{\boldsymbol{k}}, \hat{\boldsymbol{s}}, \hat{\boldsymbol{a}})$, is tied to the direction of the mean field, $\hat{\boldsymbol{b}}_{0}=\hat{\boldsymbol{z}}$, to which the local field direction, $\hat{\boldsymbol{b}}$, is inclined by an angle $\theta \sim v_{L_{\perp}} / v_{\mathrm{A}}$. Consequently, our method for spectral decomposition erroneously mixes Alfvén and slow modes. However, for nearly transverse cascades the mixing is only of the order of $\theta^{2} \ll 1$. $k_{\|}$;

Field-line tilt also causes $k_{r}$ and $k_{z}$ to differ from $k_{\perp}$ and

$$
k_{r}=\cos \theta k_{\perp}+\sin \theta k_{\|}, \quad k_{z}=-\sin \theta k_{\perp}+\cos \theta k_{\|} .
$$

Thus, $k_{r} \approx k_{\perp}\left[1+O\left(\theta^{2}\right)\right]$. However, $k_{z} \approx k_{\|}+\theta k_{\perp} \approx \theta k_{\perp}$, where the final relation applies because the degree of anisotropy increases with increasing $k_{\perp}$ along MHD cascades. Thus, $k_{\perp}$ can be represented to acceptable accuracy by $k_{r}$. However, $k_{\|}$cannot be obtained from $k_{z}$. Henceforth, we treat as equivalent $k_{r}$ and $k_{\perp}$ and $L_{x}=L_{y}$ and $L_{\perp}$. However, we are always careful to distinguish $k_{z}$ from $k_{\|}$and to note that

$$
k_{z} \approx \frac{v_{L_{\perp}}}{v_{\mathrm{A}}} k_{\perp}
$$

\subsection{Power Spectra}

Three-dimensional power spectra of field quantities, $E_{3 \mathrm{D}}(\boldsymbol{k})$, are azimuthally symmetric functions of $\boldsymbol{k}_{\boldsymbol{r}}=k_{x} \hat{\boldsymbol{x}}$ $+k_{y} \hat{y}$ at fixed $k_{z} \cdot{ }^{9}$ Accordingly, we define the twodimensional integrated power spectrum by

$$
E_{2 \mathrm{D}}\left(k_{r}, k_{z}\right)=k_{r} \int_{0}^{2 \pi} d \phi E_{3 \mathrm{D}}(\boldsymbol{k}) \text {. }
$$

It is important to note that $E_{2 \mathrm{D}}\left(k_{r}, k_{z}\right)$ is not equivalent to $E_{2 \mathrm{D}}\left(k_{\perp}, k_{\|}\right)$. Moreover, the latter cannot be derived from the former. This shortcoming is due to the failure of the spectral decomposition procedure described in $\S 4.1$ to determine $k_{\|}$. It means that the two-dimensional power spectrum is not a useful quantity. ${ }^{10}$ However, we make so much use of the one-dimensional integrated power spectrum, defined by

$$
E_{1 \mathrm{D}}\left(k_{r}\right)=\int_{-\infty}^{\infty} d k_{z} E_{2 \mathrm{D}}\left(k_{r}, k_{z}\right)
$$

that henceforth we drop the subscript 1D.

\subsection{Structure Functions}

The three-dimensional behavior of MHD turbulence is best captured in real space using second-order structure functions tied to the local magnetic field. We define transverse and longitudinal structure functions for the vector field $\boldsymbol{U}$ by

$$
\begin{aligned}
\operatorname{SFT}_{U}\left(x_{\perp}\right) \equiv & \left\langle\left[\boldsymbol{U}\left(\boldsymbol{x}^{\prime}+\boldsymbol{x}_{\perp}\right)-\boldsymbol{U}\left(\boldsymbol{x}^{\prime}\right)\right]\right. \\
& \left.\cdot\left[\boldsymbol{U}\left(\boldsymbol{x}^{\prime}+\boldsymbol{x}_{\perp}\right)-\boldsymbol{U}\left(\boldsymbol{x}^{\prime}\right)\right]\right\rangle,
\end{aligned}
$$

where $\boldsymbol{x}_{\perp} \cdot \boldsymbol{b}=0$, and

$$
\begin{aligned}
\operatorname{SFL}_{U}\left(x_{\|}\right) \equiv & \left\langle\left[\boldsymbol{U}\left(\boldsymbol{x}^{\prime}+\boldsymbol{f}\left(x_{\|}\right)\right)-\boldsymbol{U}\left(\boldsymbol{x}^{\prime}\right)\right]\right. \\
& \left.\cdot\left[\boldsymbol{U}\left(\boldsymbol{x}^{\prime}+\boldsymbol{f}\left(x_{\|}\right)\right)-\boldsymbol{U}\left(\boldsymbol{x}^{\prime}\right)\right]\right\rangle,
\end{aligned}
$$

\footnotetext{
${ }^{9}$ In any specific realization, this is true only in a statistical sense.
}

${ }^{10}$ We obtain two-dimensional information from structure functions. with $\boldsymbol{f}\left(x_{\|}\right)=\int_{0}^{x} \| d s \hat{\boldsymbol{b}}(s)$. The integral is taken along the field direction with $s$ measuring arc length from $\boldsymbol{x}^{\prime}$. Averaging over $\boldsymbol{x}^{\prime}$ is done with random volume sampling. Since the vector fields of interest possess statistical axial symmetry about $\hat{\boldsymbol{b}}$, we include an axial averaging of the direction of $\boldsymbol{x}_{\perp}$ at fixed $x_{\perp} \equiv\left|x_{\perp}\right|$ in the computation of $\operatorname{SFT}_{U}\left(x_{\perp}\right)$.

\subsection{Time Step and Hyperviscosity}

The anisotropy of MHD turbulence complicates the discussion of constraints on the time step and hyperviscosity. Accordingly, we begin by discussing the simpler case of spectral simulation of isotropic hydrodynamic turbulence. These constraints are summarized in Figure 2.

\subsubsection{Isotropic Hydrodynamic Turbulence}

We assume the Kolmogorov scaling. Given velocity $v_{L}$ on outer scale $L$, inertial-range velocity differences across $\lambda \lesssim L$ scale as $v_{\lambda} \sim(\lambda / L)^{1 / 3} v_{L}$ down to inner scale $l \sim\left(v_{n} / v_{L} L^{2 n-1}\right)^{3 /(6 n-2)} L$.

Four conditions constrain the values of the time step, $\Delta t$, and hyperviscosity, $v_{n}$, suitable for a spectral simulation of isotropic hydrodynamic turbulence. Each refers to the behavior of modes with the largest wavevectors, $k_{M}$. We express these constraints in terms of the dimensionless variables $\overline{\Delta t} \equiv v_{L} k_{M} \Delta t$ and $\bar{v} \equiv\left(v_{n} k_{M}^{2 n}\right) /\left(v_{L} k_{M}\right)$.

Conditions 1 and 2 are concerned with computational accuracy.

1. Advection by outer scale eddies gives rise to fractional changes of the order of $v_{L} k_{M} \Delta t$ in the Fourier components of the smallest scale modes during one time step. ${ }^{11}$ Accurate

\footnotetext{
${ }^{11}$ Changes caused by interactions that are local in Fourier space are smaller by a factor of $\left(k_{M} L\right)^{-1 / 3}$.
}

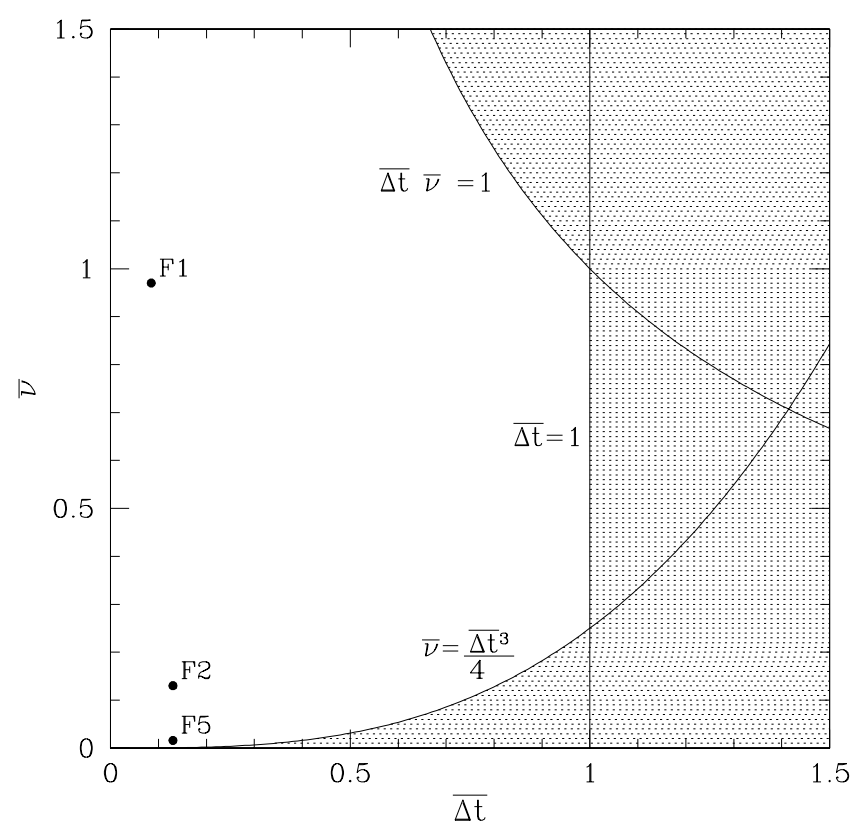

FIG. 2.-Time step and hyperviscosity. We illustrate constraints on the dimensionless time step and hyperviscosity as described in $\S 4.4$. The constraint given by eq. (68) is not shown, because it depends upon an additional parameter. Allowed choices lie in the unshaded part of the figure. Each plotted point represents values used in an individual simulation. 
computation requires

$$
\overline{\Delta t} \lesssim 1
$$

which is the spectral equivalent of the Courant condition in real space.

2. Hyperviscosity causes a fractional decay of the order of $v_{n} k_{M}^{2 n} \Delta t$ in the amplitudes of the smallest scale modes during a single time step. Thus,

$$
\overline{\Delta t} \lesssim \frac{1}{\bar{v}_{n}}
$$

Conditions 3 and 4 are required to maintain stability.

3. This constraint depends on the algorithm used to advance the variables in time. Integration with the secondorder Runge-Kutta algorithm (RK2) results in an unphysical transfer of energy from large- to small-scale modes. Consider one-dimensional uniform advection at speed $v_{L}$ of the single Fourier mode $v\left(k_{M}, t\right)$. RK2 yields $v\left(k_{M}, \Delta t\right)=\left[1-i v_{L} k_{M} \Delta t-\left(v_{L} k_{M} \Delta t\right)^{2} / 2\right] v\left(k_{M}, 0\right)$. Thus, $\left|v\left(k_{M}, \Delta t\right)\right|^{2}=\left[1+\left(v_{L} k_{M} \Delta t\right)^{4} / 4\right]\left|v\left(k_{M}, 0\right)\right|^{2}$. In order for hyperviscosity to maintain stability,

$$
\overline{\Delta t} \lesssim\left(4 \bar{v}_{n}\right)^{1 / 3} \text {. }
$$

4. A turbulent cascade transfers energy from large to small scales, where it is dissipated by viscosity. Spectral simulations of turbulence must include a mechanism that is able to dispose of the energy carried by the cascade before it reaches $k_{M}$. Otherwise, it would reflect back to smaller $k$ and the high- $k$ Fourier modes would approach energy equipartition with those of lower $k .^{12}$ Hyperviscosity suffices provided that the inner scale it sets is larger than the grid resolution. This requires

$$
\bar{v} \gtrsim\left(k_{M} L\right)^{-1 / 3}=(\pi N / 2)^{-1 / 3} .
$$

Dealiasing also involves a loss of energy and can stabilize simulations run with a sufficiently small time step even in the absence of hyperviscosity. Further investigation is needed to clarify the manner in which energy is lost due to dealiasing.

\subsubsection{Anisotropic Magnetohydrodynamic Turbulence}

We restrict our discussion of MHD turbulence to cases in which the energy in the mean magnetic field greatly exceeds that in the kinetic and magnetic fluctuations. As discussed in $\S 3$, analytic arguments indicate that the Kolmogorov scaling is obeyed in planes perpendicular to the mean magnetic field. Thus, constraints on the time step and hyperviscosity deduced in $\S 4.4 .1$ for hydrodynamic turbulence pertain to MHD turbulence in the $x y$ plane provided that we take $\overline{\Delta t} \equiv v_{L_{\perp}} k_{M_{\perp}} \Delta t$ and $\bar{v} \equiv\left(v_{n} k_{M_{\perp}}^{2 n}\right) /\left(v_{L_{\perp}} k_{M_{\perp}}\right)$.

Different constraints arise from motion along the direction of the mean magnetic field. Strong MHD turbulence is anisotropic, with energy cascading more rapidly along $k_{\perp}$ than along $k_{\|}$. Analytic arguments imply that the anisotropy at the perpendicular scale $k_{\perp}^{-1}$ is determined by the condition that the nonlinearity parameter $\chi=$ $\left(v_{\lambda} k_{\perp}\right) /\left(v_{\mathrm{A}} k_{\|}\right) \sim 1$, where $k_{\|}$is the wavevector component in lence.

12 The energy per Fourier mode scales as $k^{-11 / 3}$ in Kolmogorov turbu- the direction of the local magnetic field. It is important to maintain the distinction between $k_{\|}$and $k_{z}$. As discussed in $\S 4.1, k_{z} \approx\left(v_{L_{\perp}} / v_{\mathrm{A}}\right) k_{\perp} \sim\left(k_{\perp} L_{\perp}\right)^{1 / 3} k_{\|}$.

We control the value of $\left(v_{L_{\perp}} L_{z}\right) /\left(v_{\mathrm{A}} L_{\perp}\right)$ in our simulations. Typically, this quantity is set somewhat larger than unity in order to ensure that the largest scale structures cascade on a timescale shorter than the Alfén crossing time, $L_{z} / v_{\mathrm{A}}$. As a consequence,

$$
v_{\mathrm{A}} k_{M_{z}} \approx \frac{v_{\mathrm{A}} L_{\perp}}{v_{L_{\perp}} L_{z}} v_{L_{\perp}} k_{M_{\perp}} \lesssim v_{L_{\perp}} k_{M_{\perp}} .
$$

After this preparation, we are ready to examine the constraints placed on $\Delta t$ and $v_{n}$ by evolution in the $z$-direction.

1. Advection in the $z$-direction is dominated by propagation at the Alfvén speed, since $v_{L_{z}} \ll v_{\mathrm{A}}$ in our simulations. Thus, computational accuracy demands $v_{\mathrm{A}} k_{M_{z}} \Delta t \lesssim 1$. Since $v_{\mathrm{A}} k_{M_{z}} \lesssim v_{L_{\perp}} k_{M_{\perp}}$, this constraint is less severe than that imposed by equation (65).

2. Hyperdiffusivity is not important in the $z$-direction because $k_{M_{z}} \ll k_{M_{\perp}}$, and we use a scalar hyperdiffusivity.

3. Integration with RK2 leads to an unphysical transfer of energy from large to small scales due to advection at the Alfvén speed. Provided that equation (67) is satisfied, this does not cause any difficulty, because $v_{\mathrm{A}} k_{M_{z}} \lesssim v_{L_{\perp}} k_{M_{\perp}}$.

4. The maximum wavenumber in the $z$-direction, $k_{M_{z}}$, must be larger than that at the inner scale of the cascade. From equation (60),

$$
k_{M_{z}} L_{z} \sim\left(\frac{v_{L_{\perp}} L_{z}}{v_{\mathrm{A}} L_{\perp}}\right) k_{M_{\perp}} L_{\perp} .
$$

As mentioned above, the factor preceding $k_{M_{\perp}} L_{\perp}$ is typically larger than unity. Thus, adequate resolution along $z$ generally requires $N_{z}>N_{\perp}$.

\subsection{Simulation Design}

We carry out simulations of both forced and decaying MHD turbulence. Amplitudes of Fourier modes within 3 lattice units of the origin, normalized wavevector $|s| \leq 3$, are incremented at each time step in simulations of forced turbulence and assigned initial values in simulations of decaying turbulence. Each component of these amplitudes receives an addition of a complex number with random phase and absolute value drawn from a Boltzmann distribution with specified mean, subject to the constraint that $\boldsymbol{k} \cdot \boldsymbol{v}(\boldsymbol{k})=0$ and $\boldsymbol{k} \cdot \boldsymbol{b}(\boldsymbol{k})=0$. Thus, we are forcing both velocity and magnetic fluctuations. Forcing $v$ alone would artificially correlate the power received by $\boldsymbol{w}_{\uparrow}$ and $\boldsymbol{w}_{\downarrow}$. With our technique, fluctuations in the energy input to waves moving in opposite directions are independent.

The aspect ratio of our simulation box is chosen to match the anisotropy of the turbulence. In most of our simulations, $L_{z} \gg L_{x}=L_{y}$. We scale lengths to $L_{z}=1$ and velocities to $v_{\mathrm{A}}=1$. Thus, waves take $\Delta t=1$ to propagate the length of the box. The excitation level, set by the parameter $\left(v_{L_{\perp}} L_{z}\right) /\left(v_{\mathrm{A}} L_{\perp}\right)$, is chosen so that the longest waves cascade in less than $\Delta t=1$. An equivalent statement is that a typical field line wanders by more than $L_{\perp}$ in the transverse direction during its passage across the length $L_{z}$ of the box. This requires the excitation parameter to be somewhat larger than unity. Typical values in our simulations are of the order of 5 . We generally run our simulations for a few cross- 
ing times. Thus, nonlinear interactions are fully expressed on all scales.

Our basic procedure comes with a variety of refinements. The fields can be decomposed into their $\boldsymbol{w}_{\uparrow}$ and $\boldsymbol{w}_{\downarrow}$ components, as given by equations (55). Each of these can be further separated into shear Alfvén and slow modes according to equations (56) and (57). In this manner we can selectively input and remove waves of any type and with any direction of propagation.

\section{SIMULATION RESULTS}

Parameters of the simulations referred to in this section are listed in Table 2. Each simulation is carried out in a box of dimensions $L_{x}=L_{y}=2 \times 10^{-3}$ and $L_{z}=1$, includes an external magnetic field of unit strength aligned with the $z$-axis, and uses a fourth-order hyperviscosity. The dimensionless forcing power is denoted by $\mathscr{P}$. It is chosen so that the rms dimensionless fluctuations of $v$ and $b$ have magnitude $3 \times 10^{-3}$. These values also characterize the initial states of simulations of decaying turbulence.

\subsection{Power Spectra}

We obtain power spectra from our simulations as described in $\S 4.2$.

\subsubsection{One-Dimensional Power Spectra}

Examples of one-dimensional power spectra obtained by averaging results from three simulations (F2, F3, and F4) of resolution $128 \times 128 \times 512$ are presented in Figure 3 . Each spectrum has an inertial-range slope of approximately 1.5 . The power spectra displayed in Figure 4 come from a single simulation (F5) with resolution $256 \times 256 \times 512$. Aside from their extended inertial ranges, they look similar to those plotted in Figure 3.

\subsubsection{Two-Dimensional Power Spectra}

Figure 5 displays a sequence of one-dimensional power spectra made by taking cuts parallel to the $s_{z}$ axis across the two-dimensional power spectrum of shear Alfvén waves from simulation F5. Note that there is negligible power at the highest $s_{z}$ even for the highest $s_{\perp}$. Thus, this simulation has adequate resolution along the $z$-direction, something we verify for each of our simulations. As we emphasize in $\$ \S 4.1$ and 4.2, these cuts do not suffice to determine the structure

TABLE 2

SimUlation PARAMETERS

\begin{tabular}{|c|c|c|c|c|}
\hline ID & $\left(N_{\perp}, N_{z}\right)$ & $\Delta t$ & $v_{4}$ & Comments \\
\hline F1. & 64,256 & $4 \times 10^{-4}$ & $5 \times 10^{-37}$ & $\mathscr{P}=2 \times 10^{-5}$ \\
\hline$F 2 \ldots \ldots$ & 128,512 & $3 \times 10^{-4}$ & $5 \times 10^{-40}$ & $\mathscr{P}=2 \times 10^{-5}$ \\
\hline F3 ...... & 128,512 & $3 \times 10^{-4}$ & $5 \times 10^{-43}$ & $\mathscr{P}=2 \times 10^{-5}$ \\
\hline $\mathrm{F} 4 \ldots \ldots$ & 128,512 & $3 \times 10^{-4}$ & $5 \times 10^{-43}$ & $\mathscr{P}=2 \times 10^{-5}$ \\
\hline F5 ....... & 256,512 & $1.5 \times 10^{-4}$ & $5 \times 10^{-43}$ & $\mathscr{P}=2 \times 10^{-5}$ \\
\hline D1 ...... & 64,256 & $4 \times 10^{-4}$ & $5 \times 10^{-40}$ & $\mathscr{P}=0$ \\
\hline $\mathrm{D} 2 \ldots \ldots$ & 64,256 & $4 \times 10^{-4}$ & $5 \times 10^{-37}$ & $\mathscr{P}=0, A_{\uparrow}+S_{\downarrow}$ \\
\hline D3 ...... & 64,256 & $4 \times 10^{-4}$ & $5 \times 10^{-37}$ & $\mathscr{P}=0, A_{\uparrow}+A_{\downarrow}$ \\
\hline D4 ...... & 128,512 & $3 \times 10^{-4}$ & $5 \times 10^{-40}$ & $2<s_{\perp}<4$ \\
\hline D5 ...... & 128,512 & $3 \times 10^{-4}$ & $5 \times 10^{-40}$ & $4<s_{\perp}<8$ \\
\hline D6...... & 128,512 & $3 \times 10^{-4}$ & $5 \times 10^{-40}$ & $8<s_{\perp}<16$ \\
\hline D7 ...... & 128,512 & $3 \times 10^{-4}$ & $5 \times 10^{-40}$ & $16<s_{\perp}<32$ \\
\hline D8 ....... & 256,512 & $1.5 \times 10^{-4}$ & $5 \times 10^{-43}$ & $16<s_{\perp}<32$ \\
\hline D9...... & 256,512 & $1.5 \times 10^{-4}$ & $5 \times 10^{-43}$ & $32<s_{\perp}<64$ \\
\hline
\end{tabular}

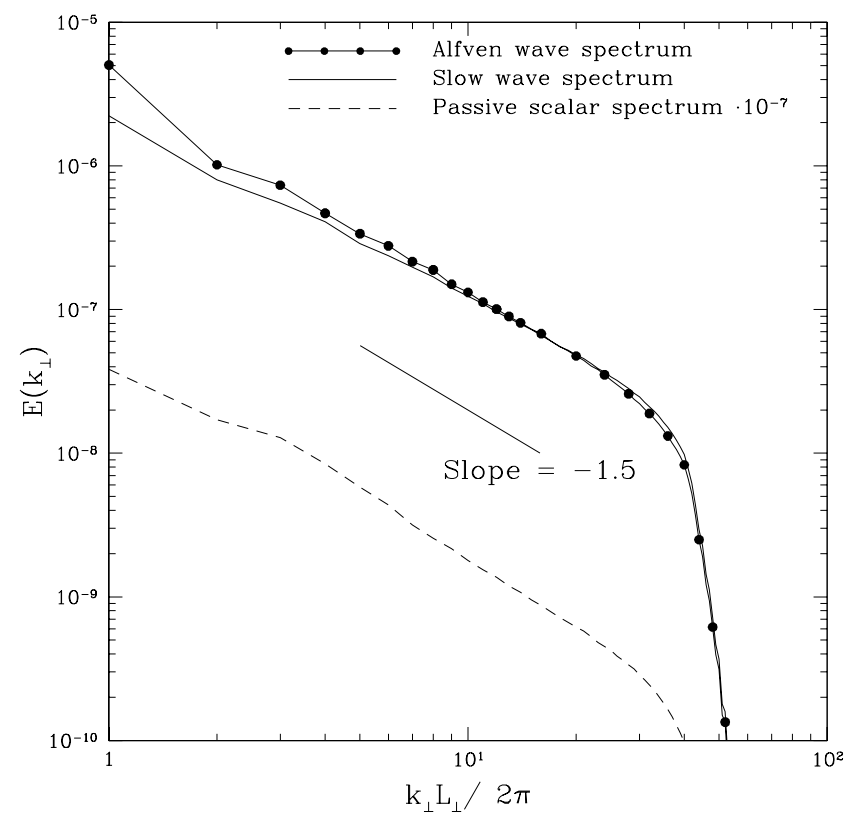

FIG. 3.-One-dimensional averaged power spectra. Energy spectra are obtained by averaging results from simulations F2, F3, and F4 with resolution $128 \times 128 \times 512$.

of turbulence parallel to the local magnetic field. For that we need to use structure functions (see $\S 5.2$ ).

\subsection{Structure Functions}

Figure 6 displays transverse and longitudinal structure functions for both shear Alfvén and slow waves calculated as described in $\S 4.3$ from data obtained by averaging results from simulations F2, F3, and F4. The plots are truncated at $\lambda / L=0.5$ because at greater separations the structure functions are affected by the application of periodic boundary conditions.

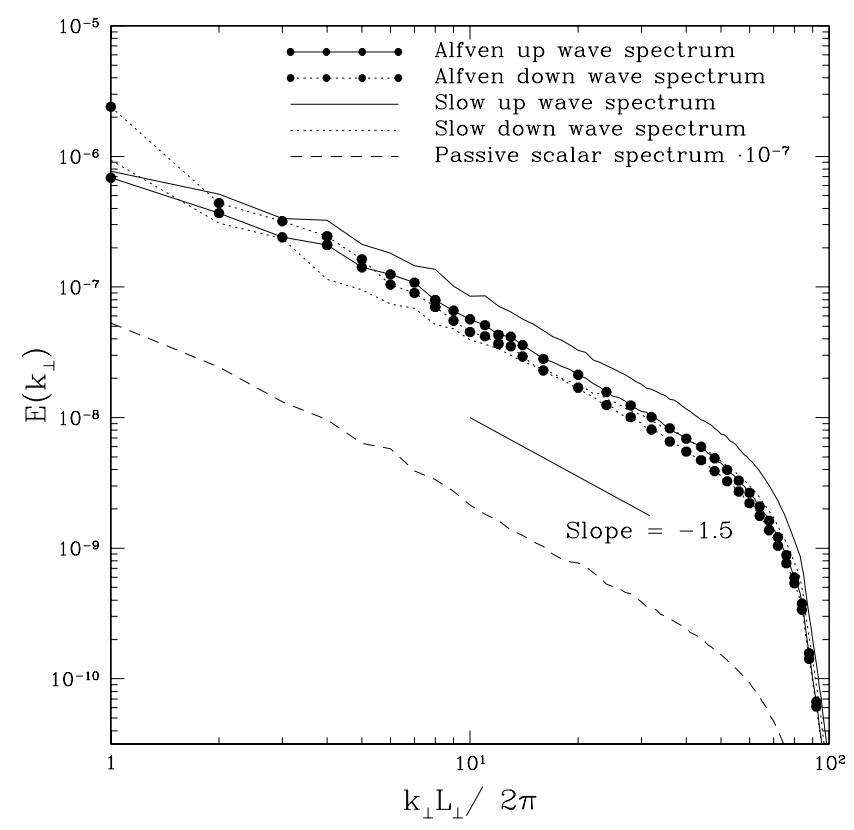

FIG. 4.- - Highest resolution one-dimensional power spectra. Energy spectra are obtained from simulation F5 with resolution $256 \times 256 \times 512$. 


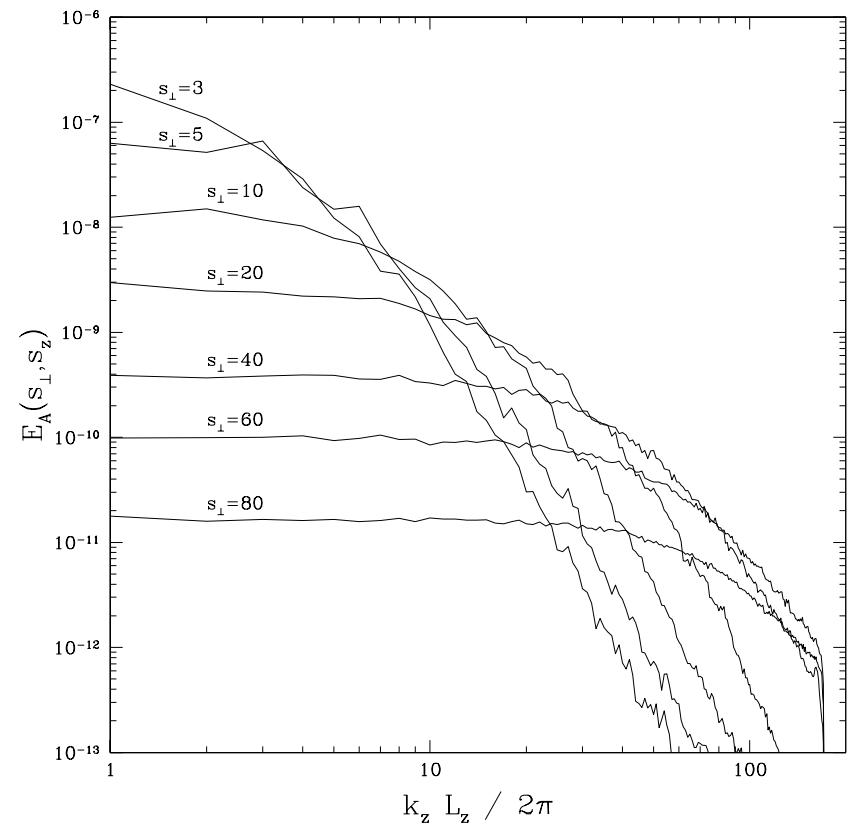

Fig. 5.-Cuts across two-dimensional power spectrum; Alfvén energy spectrum as a function of $s_{z}$ at fixed $s_{\perp}$ from simulation F5.

\subsubsection{Anisotropy}

Structure functions are the best measure of the scaledependent anisotropy of an MHD cascade. Ordered pairs of $\lambda_{\|}$and $\lambda_{\perp}$ obtained by equating the longitudinal and transverse structure functions for shear Alfvén waves shown in Figure 6 are plotted in Figure 7. We leave it to the reader to judge the degree to which this supports the prediction by GS that $\lambda_{\|} \propto \lambda_{\perp}^{2 / 3}$ in the inertial range.

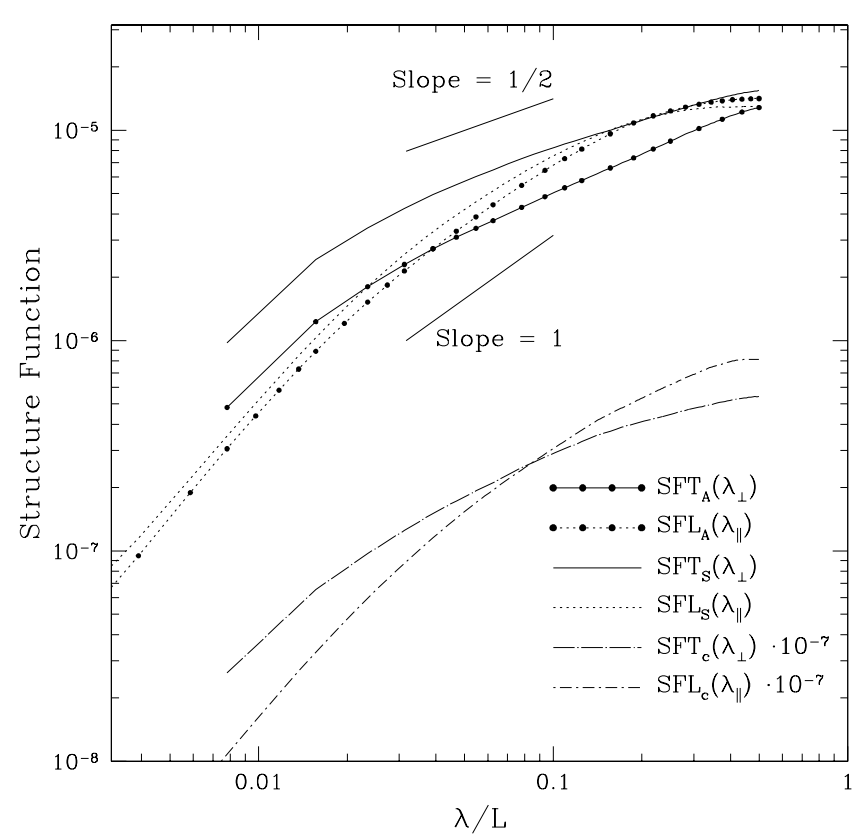

FIG. 6.-Transverse and longitudinal structure functions. Structure functions transverse and longitudinal to the local magnetic field direction are obtained by averaging results from simulations F2, F3, and F4 with resolution $128 \times 128 \times 512$.

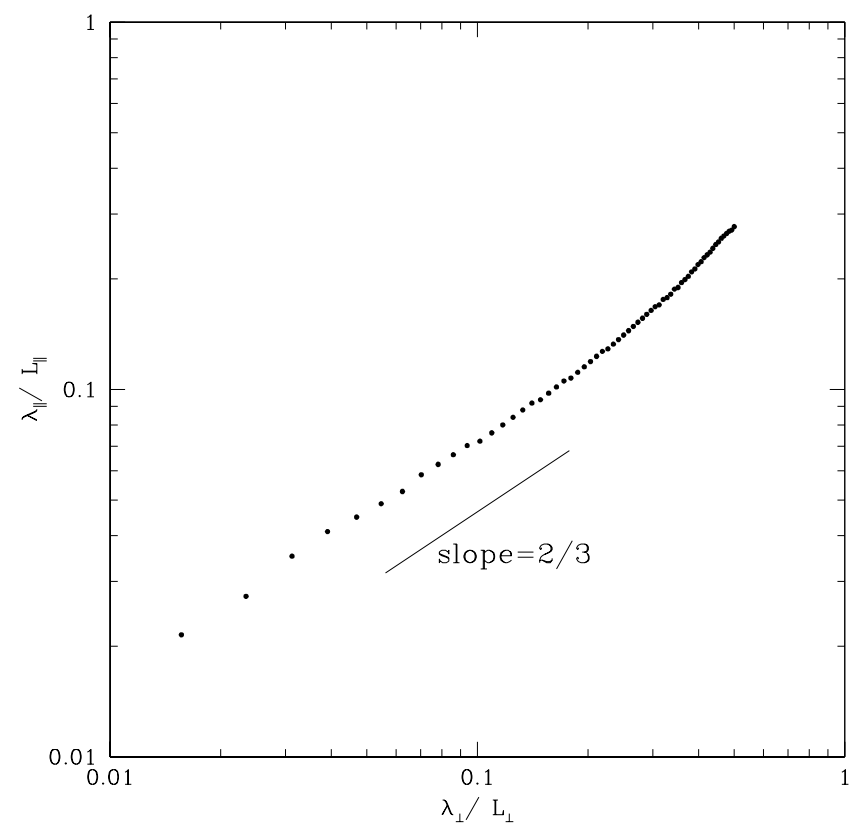

FIG. 7.-Ordered pairs of $\lambda_{\perp}$ and $\lambda_{\|}$. Anisotropy of MHD turbulence is quantified by plotting values of $\lambda_{\perp}$ and $\lambda_{\|}$obtained by setting $\operatorname{SFT}_{A}\left(\lambda_{\perp}\right)=$ $\operatorname{SFL}_{\mathbf{A}}\left(\lambda_{\|}\right)$using the data displayed in Fig. 6.

\subsubsection{Ratio of Nonlinear to Linear Timescales}

The quantity $\chi=\left(\lambda_{\perp} v_{\mathrm{A}}\right) /\left(\lambda_{\|} v_{\lambda_{\perp}}\right)$ is the ratio of the nonlinear to linear timescale associated with wave packets of dimensions $\left(\lambda_{\perp}, \lambda_{\|}\right)$. To evaluate $\chi$ we take $v_{\lambda_{\perp}}=\operatorname{SFT}_{\mathrm{A}}^{1 / 2}\left(\lambda_{\perp}\right)$ from Figure 6 and $\lambda_{\perp} / \lambda_{\|}$from Figure 7. The plot in Figure 8 establishes that $\chi$ maintains a value near unity throughout the inertial range.

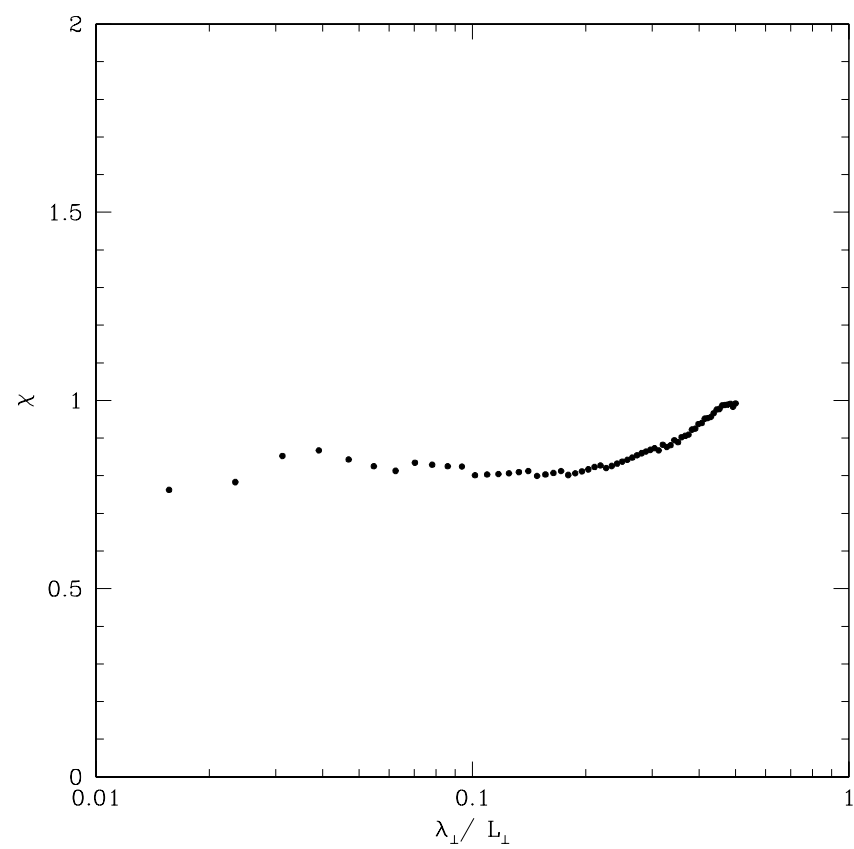

Fig. 8.-Critical balance. Data from Figs. 6 and 7 are combined to form $\chi$, the ratio of linear to nonlinear timescales. Note that $\chi$ has a nearly constant value close to unity throughout the inertial range. This confirms that MHD turbulence maintains a state of critical balance. 


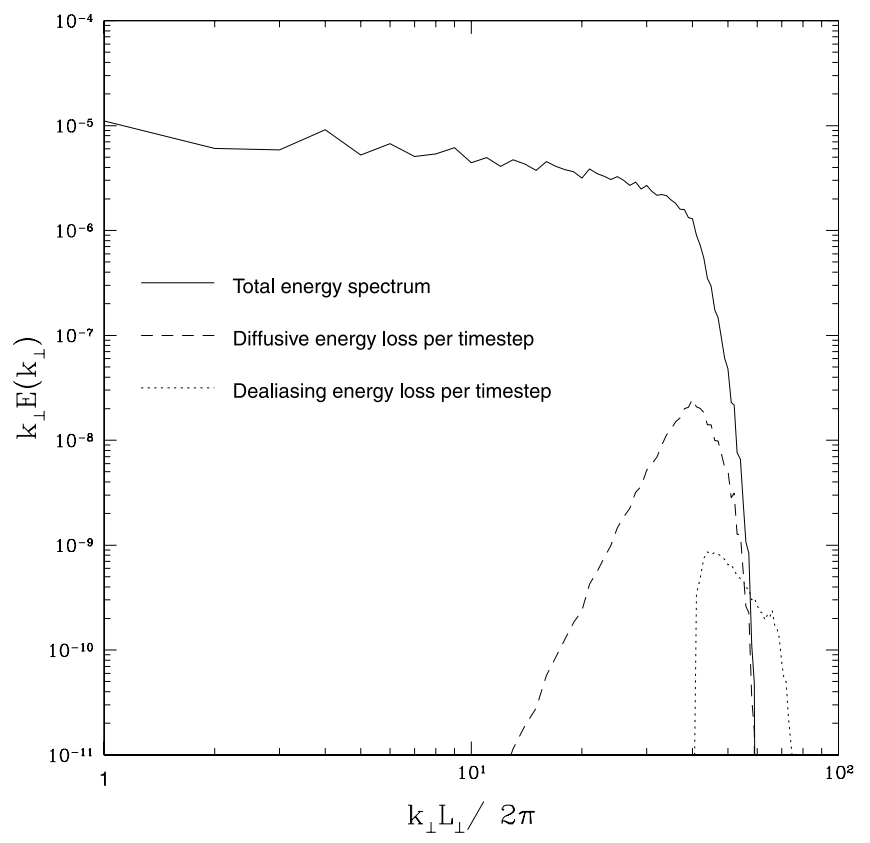

FIG. 9.-Energy loss per time step by hyperviscous dissipation and dealiasing. Energy is lost from high- $k_{\perp}$ modes by hyperviscous dissipation and dealiasing. The former dominates the latter when integrated over the spectrum. Neither is significant in the inertial range. The results shown here are from simulation $\mathrm{F} 2$.

\subsection{Energy Loss}

Total, mechanical plus magnetic, energy is conserved in the inertial range of MHD turbulence. It is lost from high$k_{\perp}$ modes by a combination of hyperviscous dissipation and dealiasing. ${ }^{13}$ Neither represents reality, but we hope that their effects do not compromise inertial-range dynamics. Figure 9 includes plots from simulation F2 of the hyperviscous and dealiasing energy losses per computational time step. For reference, the total power spectrum is also displayed. Hyperviscous dissipation dominates dealiasing except at the highest $k_{\perp}$, where the residual power is negligible. This situation is typical of all our simulations.

\subsection{Imbalance}

Because only oppositely directed waves interact, turbulent cascades tend to become unbalanced. By unbalanced, we mean that unequal fluxes of energy propagate in opposite directions along the magnetic field.

\subsubsection{Forced Turbulence}

Mode energies from simulation F1 of forced turbulence with resolution $64 \times 64 \times 256$ are plotted as a function of time in Figure 10. ${ }^{14}$ Characteristic fluctuations of order unity occur on a timescale $\Delta t=1$. Imbalance appears to saturate on longer timescales.

\subsubsection{Decaying Turbulence}

Imbalance is more severe in decaying turbulence. Figure 11 displays energies of individual modes as a function of time obtained from simulation D1 of resolution $64 \times 64 \times 256$. The initial imbalance increases without limit.

${ }^{13}$ Energy is lost during dealiasing when we set the amplitudes of modes with $\left|s_{\alpha}\right|>N_{\alpha} / 3$ to zero.

${ }_{14}$ This simulation is the source of initial conditions for many higher resolution simulations.

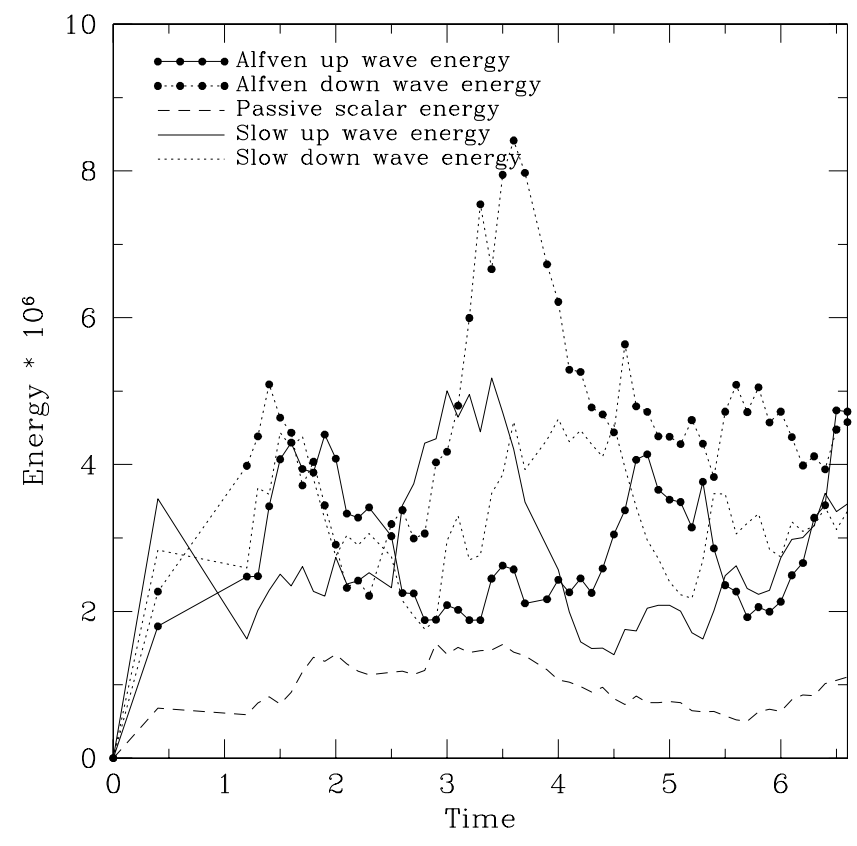

FIG. 10.-Forced turbulence: mode energies as a function of time for forced turbulence from simulation F1 of resolution $64 \times 64 \times 256$.

\subsection{Passive Role of Slow Waves}

\subsubsection{Cascading of Slow Waves by Shear Alfvén Waves}

Simulation D2 of decaying turbulence with resolution $64 \times 64 \times 256$ is designed to assess the mutual effects of shear Alfvén waves on slow waves and vice versa. We initialize it by removing the upward-propagating slow waves and the downward-propagating shear Alfvén waves from simulation $\mathrm{F} 1$ at $t=6.6$. It is then run for $\Delta t=1$. Figure 12 illustrates the evolution of the energies in upward-

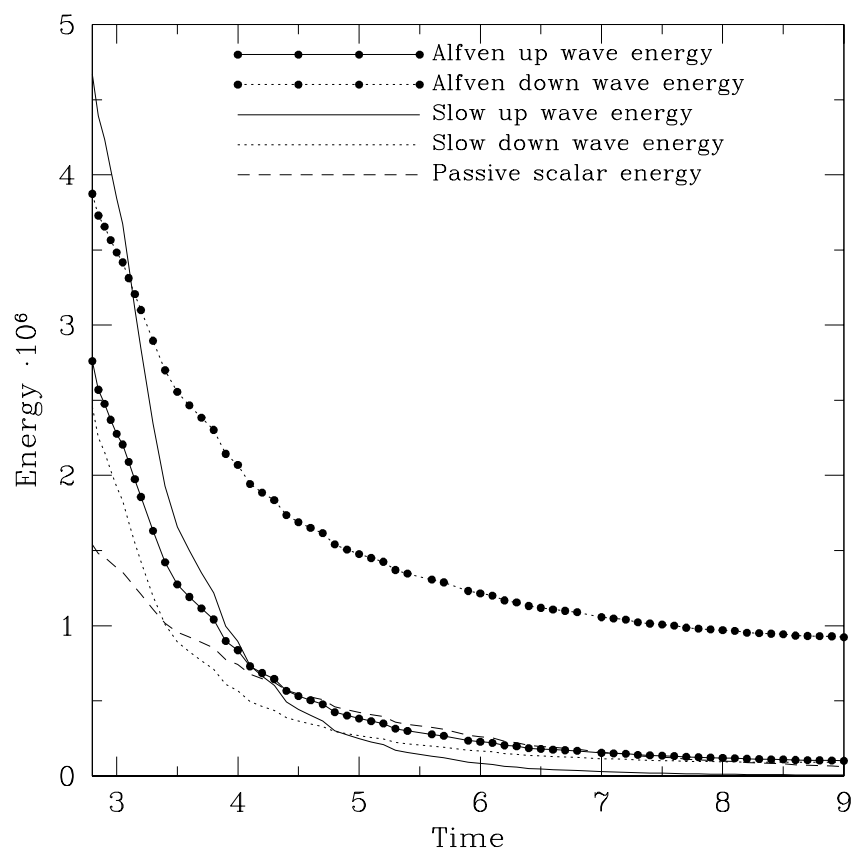

FIG. 11.-Decaying turbulence: energy as a function of time for shear Alfvén and slow modes in decaying turbulence. The simulation is D1 with resolution $64 \times 64 \times 256$. 


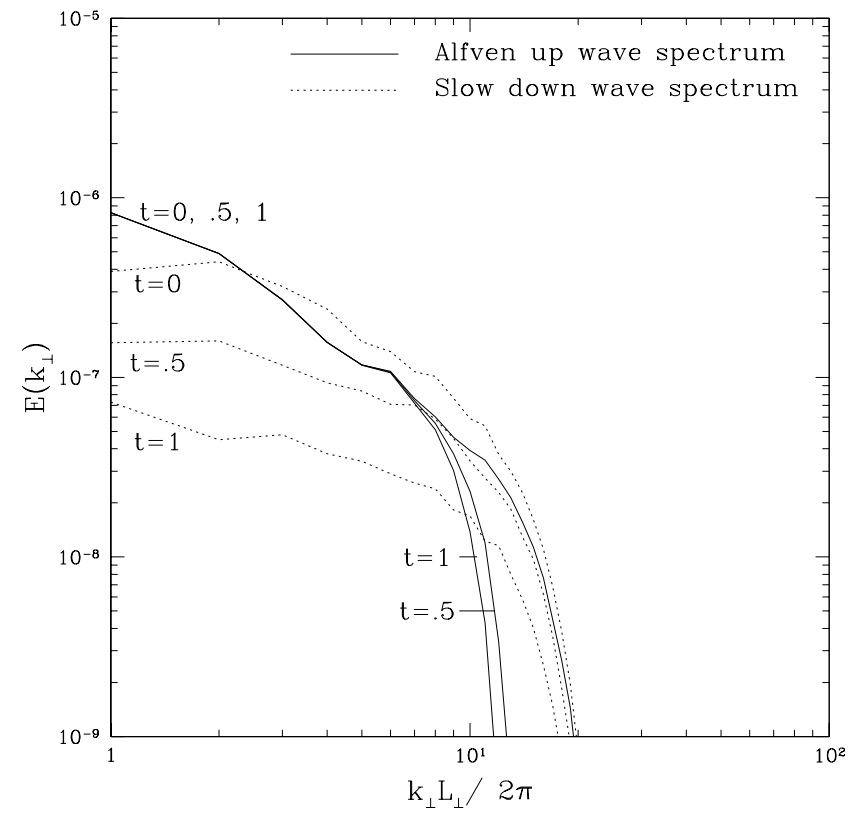

FIG. 12.-Passive role of slow waves. Upward-moving shear Alfvén waves interact with downward-moving slow waves. The power spectrum of the former decays only at large $k_{\downarrow}$, whereas that of the latter decays at all $k_{\perp}$. Results are taken from simulation D2 of decaying turbulence with resolution $64 \times 64 \times 256$.

propagating shear Alfvén waves and downwardpropagating slow waves. The only change in the spectrum of shear Alfvén waves is a decay at large $k_{\perp}$, which is entirely attributable to energy loss by hyperviscosity and dealiasing. By contrast, the spectrum of slow waves decays at all $k_{\perp}$ at a rate consistent with that shown in Figure 11. These findings demonstrate that shear Alfvén waves control the MHD cascade and that the slow waves play a passive role.

\subsubsection{Conversion of Shear Alfvén Waves to Slow Waves}

Simulation D3 of decaying turbulence with resolution $64 \times 64 \times 256$ is tailored to measure the rate at which shear Alfvén waves are converted into slow waves. It is initialized from $\mathrm{F} 1$ at $t=6.6$ by removing all slow waves and then run for $\Delta t=1$. As demonstrated by Figure 13, at the end of this interval, which corresponds to about a decay time at the outer scale (see Fig. 11), the slow waves carry negligible energy. The small admixture shown may result from the limited ability of our scheme of spectral decomposition to distinguish slow waves from shear Alfvén waves, as discussed in $\S 4.1$.

\subsection{Cascade Diagnostics}

We design special simulations to exploit the fact that only oppositely directed waves interact. Each of these is initialized by removing all but a narrow band in $k_{\perp}$ of upwardpropagating waves (up-waves) from a fully developed forced simulation. These are then run without forcing so that we can observe the evolution of the energy in the up-band as it spreads into adjacent bands. Since the down-waves evolve weakly, we restrict the lengths of these runs to $\Delta t=1 / 2$ so that interactions do not repeat.

Initial conditions for simulations D4, D5, D6, and D7 are provided by band-filtering simulation $\mathrm{F} 2$ at $t=2.8$ with up-modes retained from $2 \leq s_{\perp} \leq 4,4 \leq s_{\perp} \leq 8,8 \leq s_{\perp} \leq$ 16 , and $16 \leq s_{\perp} \leq 32$, respectively. Each of these simula-

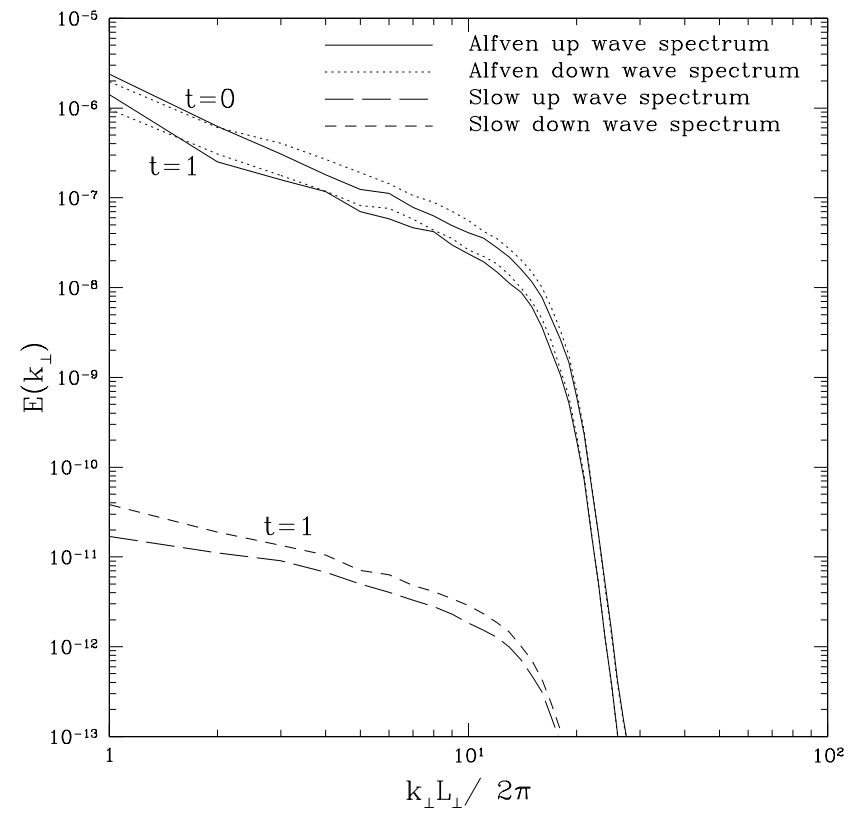

FIG. 13.-Negligible conversion of shear Alfvén to slow waves. Slow wave production in a simulation of decaying turbulence initialized with pure shear Alfvén waves. After one decay time, the slow waves contain $\lesssim 10^{-4}$ of the total energy. This amount is indistinguishable from the false slow waves that our spectral decomposition procedure would report because of the tilt of the local magnetic field relative to the global $z$-axis. Data plotted comes from simulation D3 with resolution $64 \times 64 \times 256$. Simulation F1 provides the initial conditions for simulation D3.

tions has resolution $128 \times 128 \times 512$. Resolution $256 \times 256 \times 512$ simulations D8 and D9 are initialized from simulation $\mathrm{F} 5$ by band-filtering at $t=2.95$ with upmodes retained from $16 \leq s_{\perp} \leq 32$ and $32 \leq s_{\perp} \leq 64$, respectively.

\subsubsection{Absence of an Inverse Cascade}

Figure 14 summarizes how energy spreads from each selected band into adjacent bands. It establishes that the predominant movement is toward higher $k_{\perp}$. There is no evidence for an inverse cascade. A more detailed demonstration for the selected band $8 \leq s_{\perp} \leq 16$ is provided in Figure 15, which is based on simulation D6.

\subsubsection{Resolution Dependence}

Figure 16 compares results from simulation D7 of resolution $128 \times 128 \times 512$ with those from simulation D8 of resolution $256 \times 256 \times 512$. Each simulation is initialized with energy in up-waves confined to the band $16 \leq$ $s_{\perp} \leq 32$. Note how well the energies in the central and lefthand bands from the two simulations match as they evolve. This establishes that the largest $k_{\perp}$ band in simulation D7 is a valid part of the inertial range.

\subsubsection{Cascade Time}

As a standard measure of the timescale for energy transfer across $\lambda_{\perp}$, we take $t_{c} \sim \lambda_{\perp} / v_{\lambda_{\perp}}$, where $v_{\lambda_{\perp}}$ is obtained from the transverse structure functions of downwardpropagating Alfvén waves according to $2 v_{\lambda_{\perp}}^{2}=\operatorname{SFT}_{\mathrm{A} \downarrow}\left(\lambda_{\perp}\right)$. Banded simulations also permit a more direct measure of the cascade time as that at which the energy in the righthand band matches that in the central band. We identify this version by the symbol $t_{h}$.

Values for the different types of cascade time are given in Table 3 . Even for the lowest $k_{\perp}$ band, each is substantially 


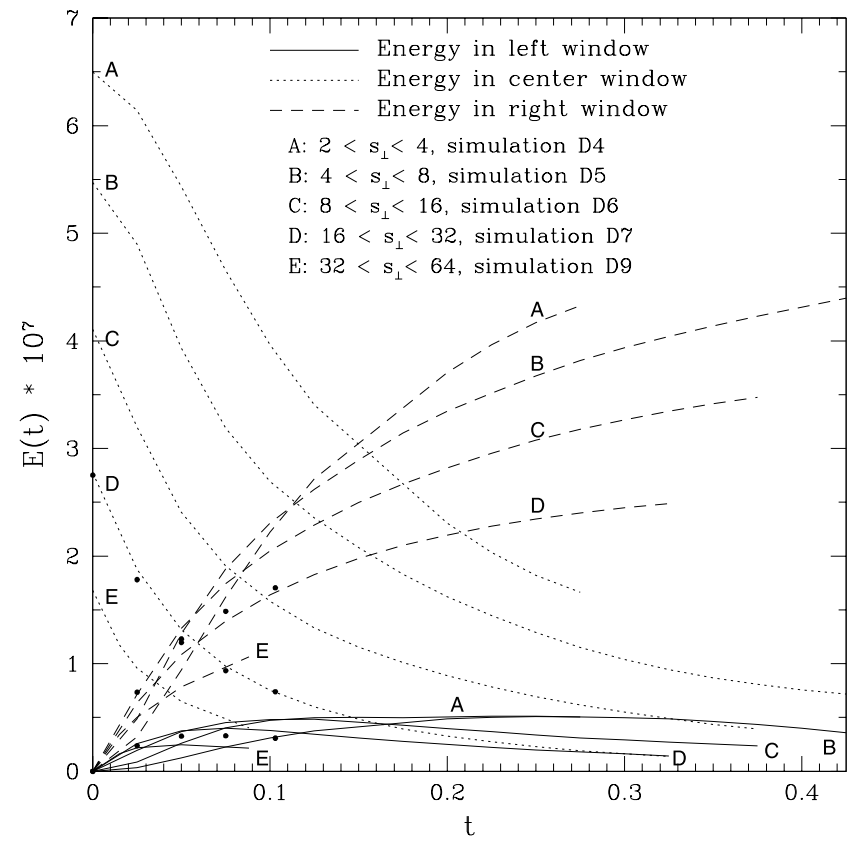

FIG. 14.- Summary of bandpass-filtered simulations. We plot the energy as a function of time in the center band and in the bands immediately to its left and right for each bandpass-filtered simulation. Initially, all the energy is in the central band. As time passes it spreads into adjacent bands. Points correspond to simulation D8, which is initialized with the same up-mode band as simulation D7, but with twice the transverse resolution in the down modes. There is good agreement between simulations D7 and D8, as is shown in more detail in Fig. 16.

smaller than the time, $\Delta t=1$, that waves take to cross the computational box.

For ease of comparison, we plot the tabulated values against $k_{\perp} L_{\perp} /(2 \pi)$ in Figure 17. Note that $t_{h}$ declines much more slowly with increasing $k_{\perp}$ than does $t_{c}$.

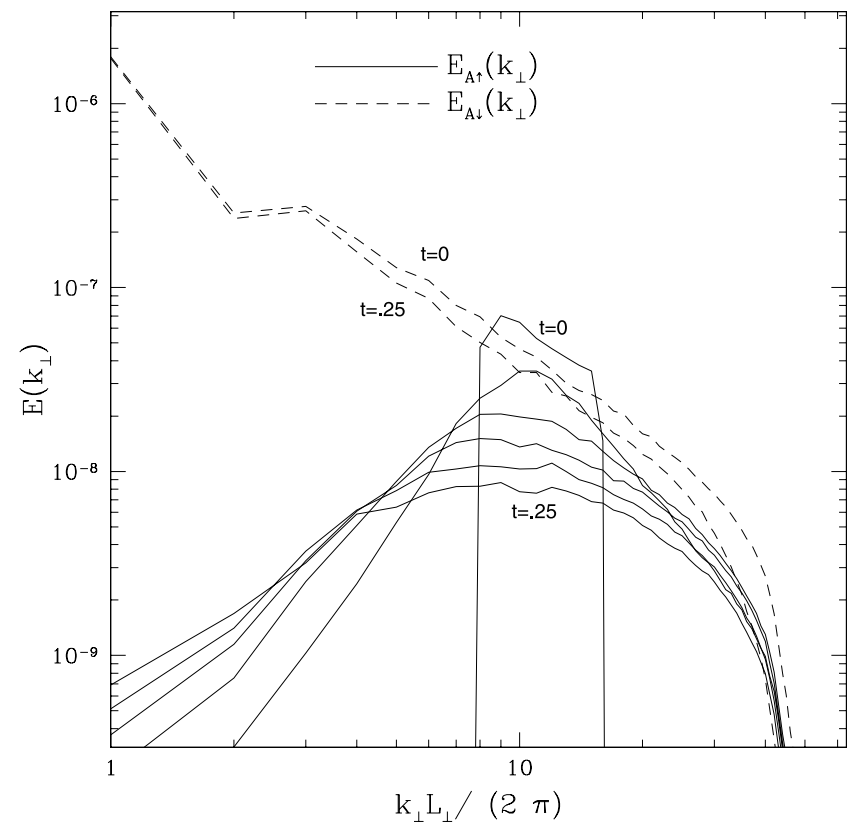

FIG. 15.-Absence of an inverse cascade. Dashed lines depict the initial and final down-wave spectra. The up-wave spectra are plotted at a succession of times differing by $\Delta t=0.05$. Almost all the energy that leaves the band $8 \leq s_{\perp} \leq 16$ moves to higher $s_{\perp}$. These data are taken from simulation D6.

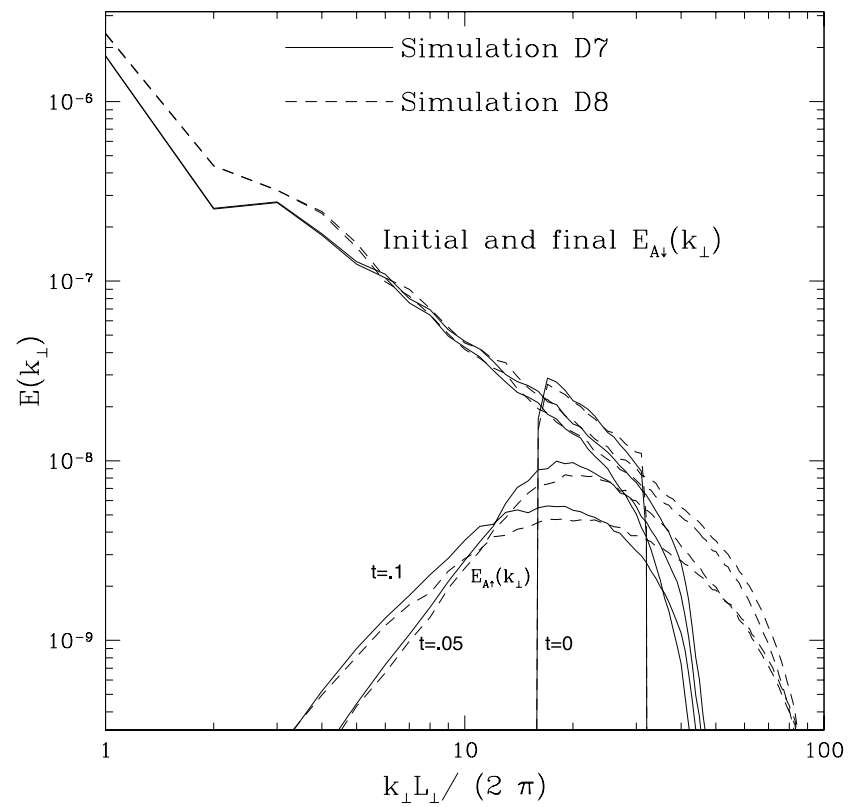

FIG. 16.-Comparison of simulations at different resolutions. Increased resolution has little effect on the evolution of energy in the left-hand and central bands. Thus, the latter resides in the inertial range in even the lower resolution simulation. Energy that moves into the right-hand band is more rapidly dissipated in the lower resolution simulation and more effectively stored in the higher resolution one.

TABLE 3

CASCADE Times

\begin{tabular}{cllccc}
\hline \hline \multicolumn{1}{c}{$s_{\perp}$} & $\lambda_{\perp} / L_{\perp}$ & \multicolumn{1}{c}{$v_{\perp}$} & $t_{c}=\lambda_{\perp} / v_{\perp}$ & $t_{h}$ & $t_{h} / t_{c}$ \\
\hline $2-4 \ldots \ldots$. & 0.188 & $3.28 \times 10^{-3}$ & 0.115 & 0.152 & 1.32 \\
$4-8 \ldots \ldots \ldots$ & 0.094 & $2.67 \times 10^{-3}$ & 0.070 & 0.115 & 1.64 \\
$8-16 \ldots \ldots$. & 0.047 & $2.10 \times 10^{-3}$ & 0.045 & 0.080 & 1.78 \\
$16-32 \ldots \ldots$ & 0.023 & $1.58 \times 10^{-3}$ & 0.029 & 0.058 & 2.00 \\
$32-64 \ldots \ldots$ & 0.0117 & $1.29 \times 10^{-3}$ & 0.0181 & 0.044 & 2.43 \\
\hline
\end{tabular}

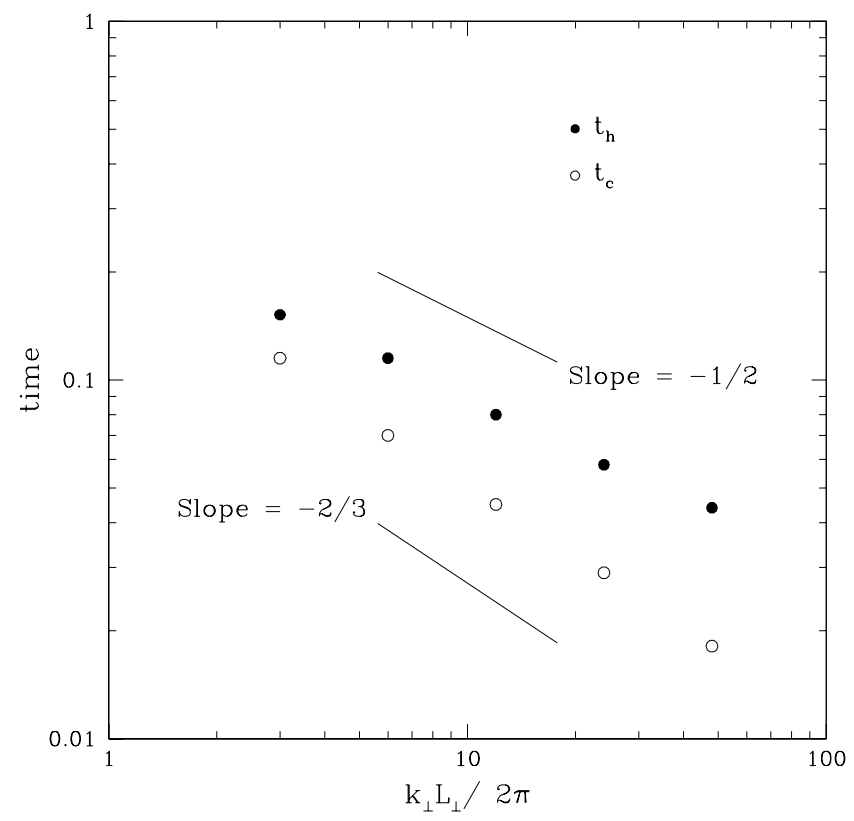

FIG. 17.-Cascade times: comparison of cascade times based on different definitions. See text for details. 

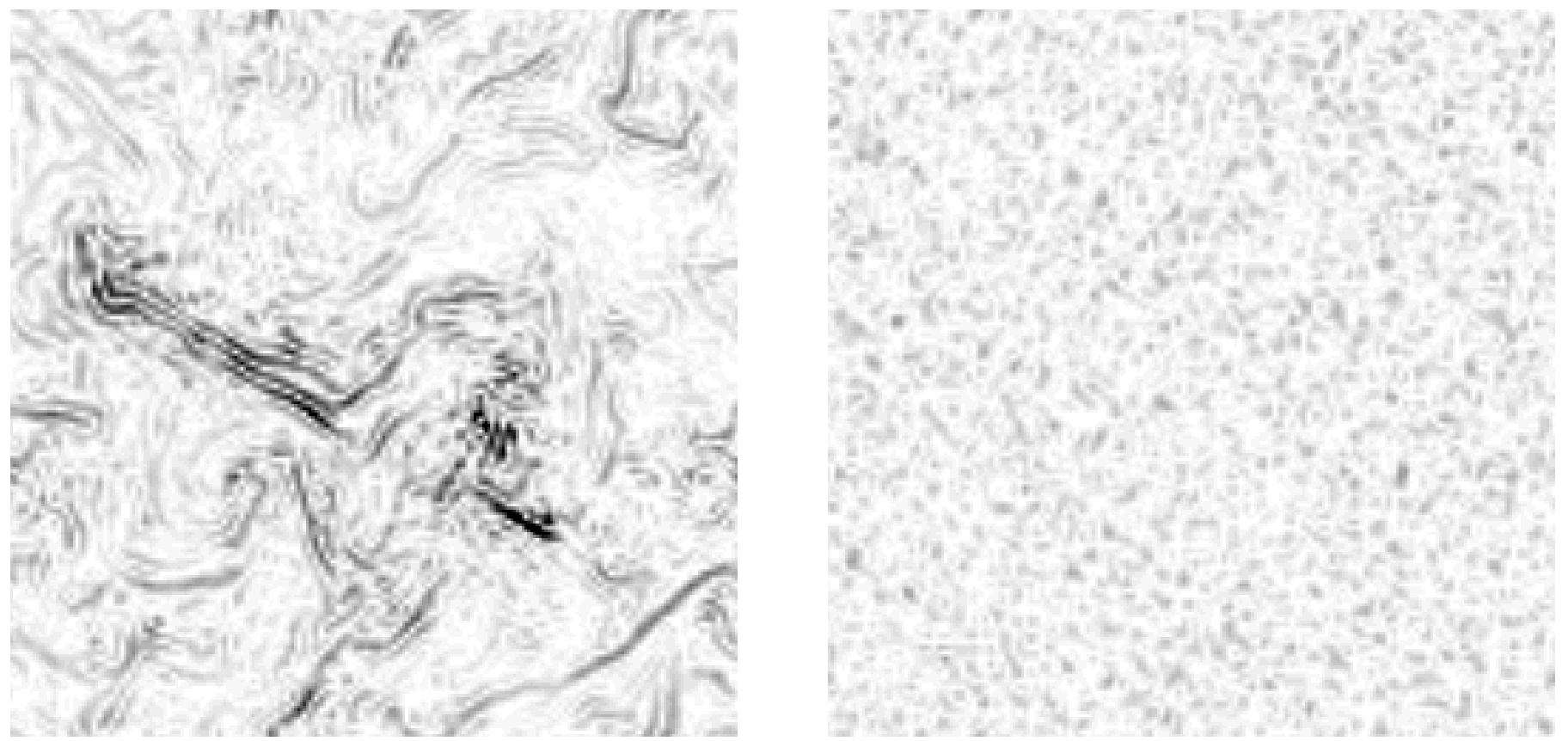

Fig. 18.-Alfvén up-modes. Left: Gray-scale image of $\left|\boldsymbol{\nabla} \times \boldsymbol{A}_{\uparrow}\right|$ in a $(x, y)$ slice at $z=0$. Right: Image based on the same Fourier coefficients with random phases, shown for comparison.

\subsection{Intermittency}

Simulations of hydrodynamic turbulence exhibit structure that is not seen in random phase realizations of velocity fields with identical power spectra (Jimenez, Wray, \& Saffman 1993). We find the same to be true for MHD turbulence. This is illustrated in Figures 18-21. The left panels display magnitudes of the curls of upward- and downwardpropagating shear Alfvén and slow waves in a $(x, y)$ slice at $z=0$ taken from simulation F5. Randomizing the phases of the Fourier coefficients used to generate the left panels yields the images shown in the right panels. Coherent structures, which are conspicuous in the former, are absent in the latter.

While the eye does an excellent job of recognizing intermittency, it is helpful to have a quantitative measure. To accomplish this, we apply a sequence of high-pass filters to the Fourier coefficients of the Elsasser fields and a sequence of low-pass filters to their gradients. A filter is identified by a
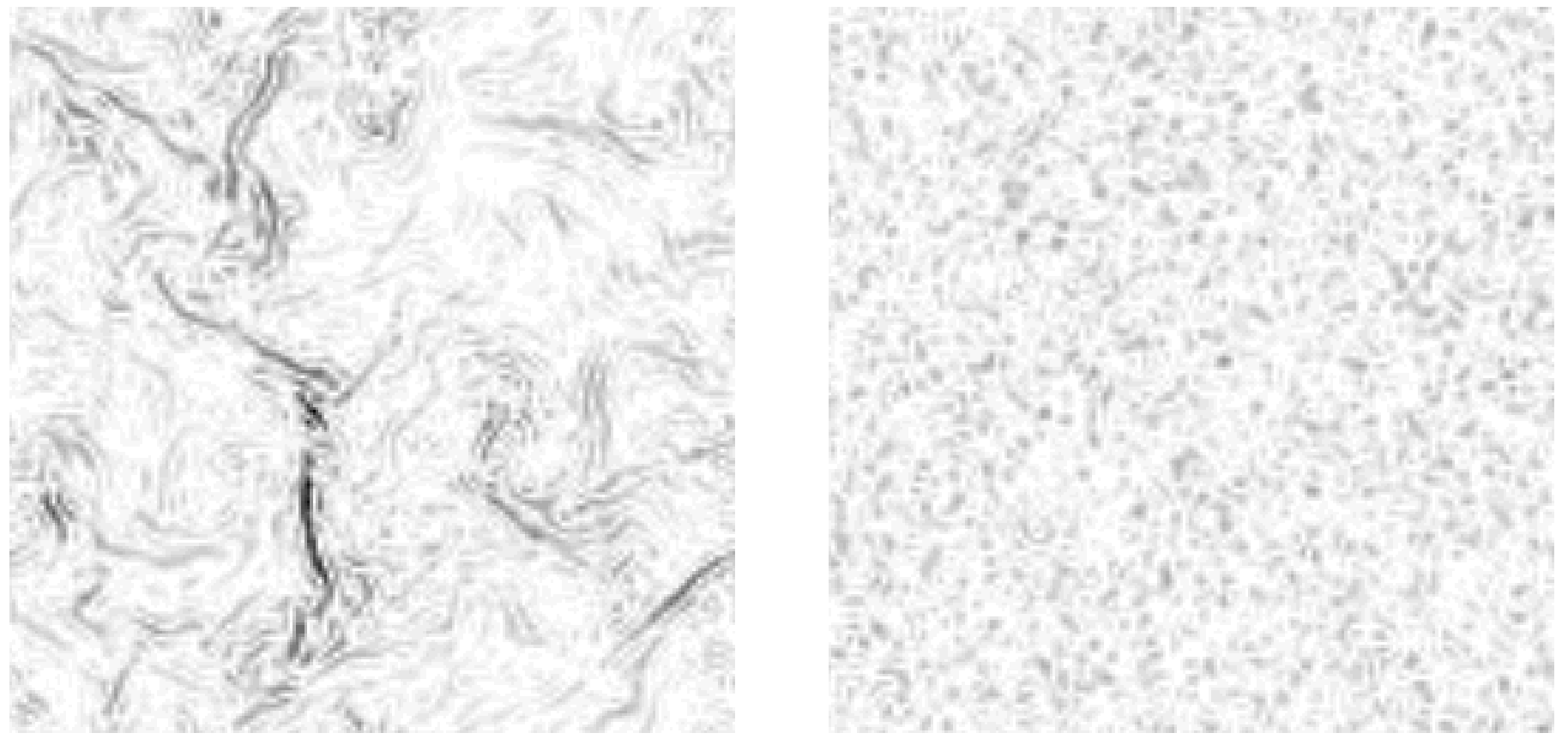

Fig. 19.-Alfvén down-modes. Left: Gray-scale image of $\left|\nabla \times \boldsymbol{A}_{\downarrow}\right|$ in a $(x, y)$ slice at $z=0$. Right: Image based on the same Fourier coefficients with random phases, shown for comparison. 

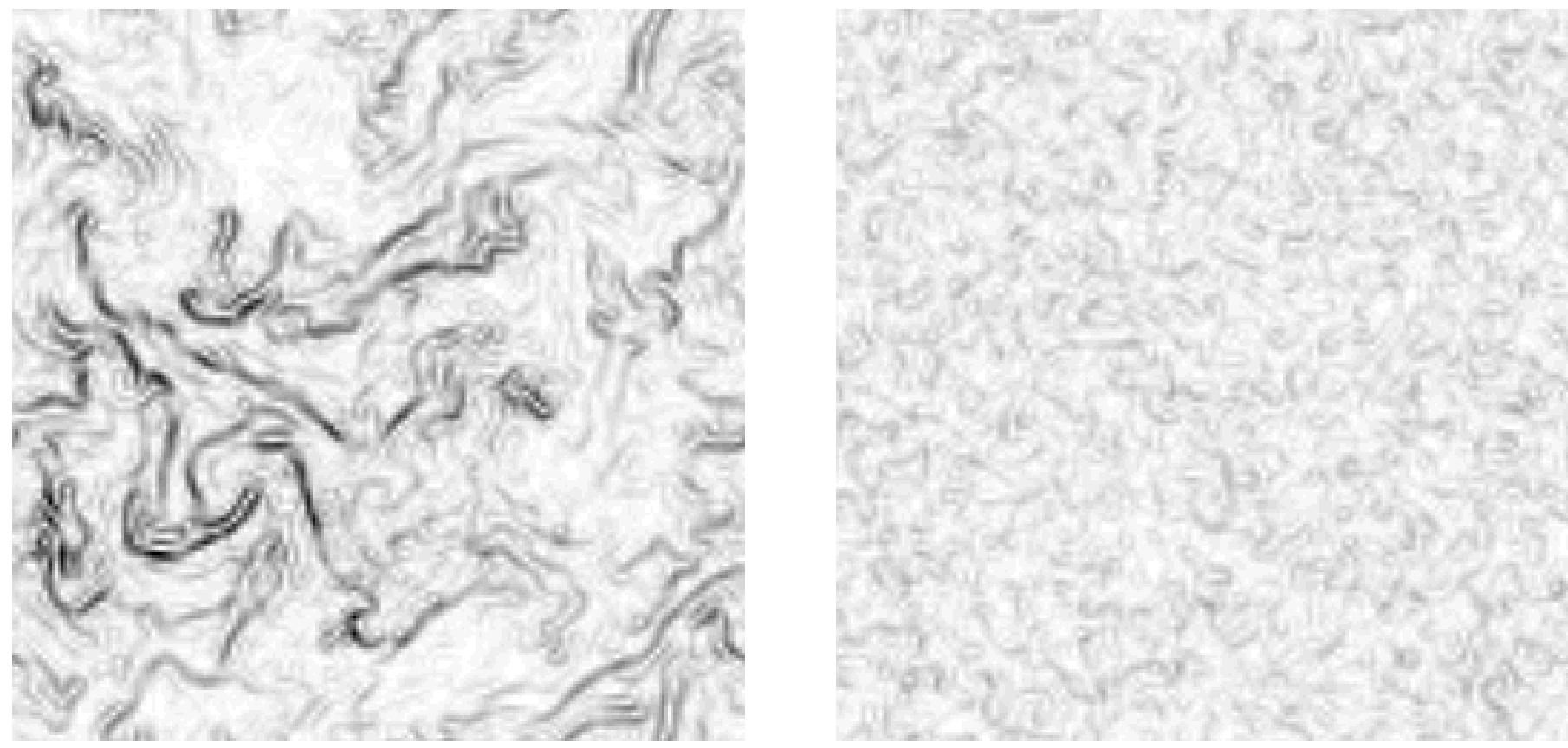

Fig. 20.-Slow up-modes. Left: Gray-scale image of $\left|\boldsymbol{\nabla} \times \boldsymbol{S}_{\uparrow}\right|$ in a $(x, y)$ slice at $z=0$. Right: Image based on the same Fourier coefficients with random phases, shown for comparison.

value of $s_{\perp}$. High-pass filters remove modes with smaller $s_{\perp}$ and low-pass filters remove those with larger $s_{\perp}$. Transverse structure in the Elsasser fields is dominated by low $s_{\perp}$ modes and that in their gradients by high $s_{\perp}$ modes. Applying a sequence of high-pass filters with increasing $s_{\perp}$ to the Fourier coefficients of the Elsasser fields emphasizes structure of decreasing scale. Similarly, applying a sequence of low-pass filters with increasing $s_{\perp}$ to the Fourier coefficients of the gradients of the Elsasser fields targets structure of increasing scale.

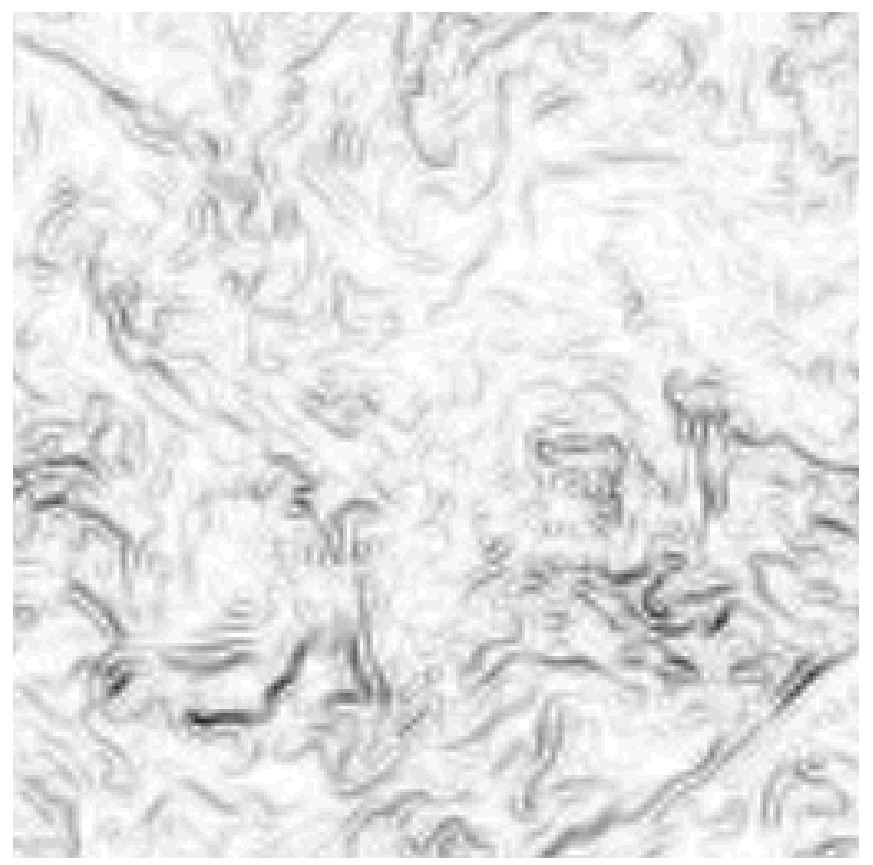

Filtered data is obtained from the simulations used to produce Figures 18-21. Normalized fourth-order moments of relevant quantities $q$ are computed according to

$$
M_{4}(q)=\frac{\left\langle q^{4}\right\rangle}{\left\langle q^{2}\right\rangle^{2}},
$$

where angle brackets denote volume average.

Figure 22 displays moments of the Elsasser fields as a function $k_{\perp}$ for high-pass-filtered data. Moments of gra-

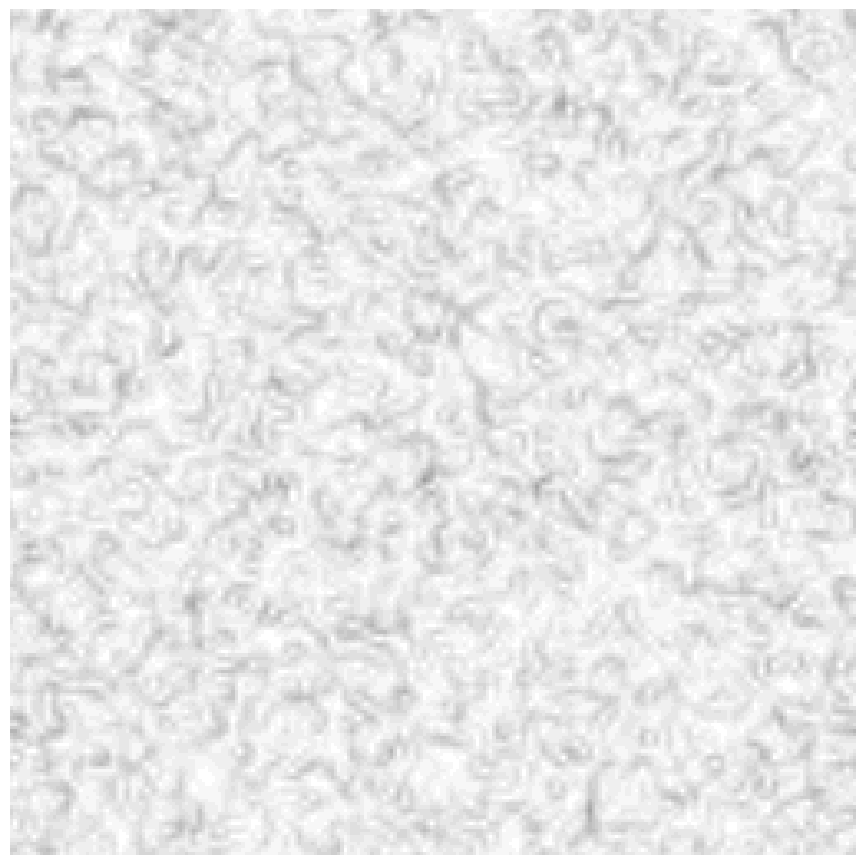

FIG. 21.-Slow down-modes. Left: Gray-scale image of $\left|\nabla \times S_{\downarrow}\right|$ in a $(x, y)$ slice at $z=0$. Right: Image based on the same Fourier coefficients with random phases, shown for comparison. 


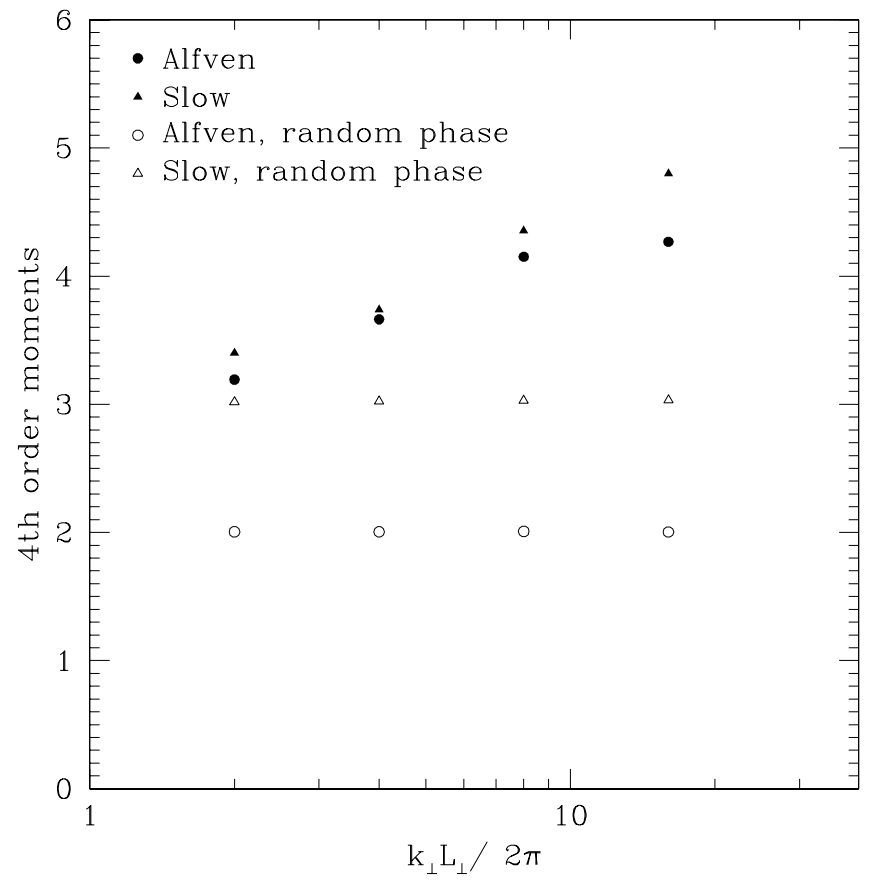

FIG. 22.-Normalized fourth-order moments for Alfvén and slow modes. Plotted points show average values of moments obtained from upward- and downward-propagating waves. The location of the high-pass filter is denoted by $k_{\perp}$.

dients of the Elsasser fields as a function of $k_{\perp}$ for low-passfiltered data are plotted in Figure 23. For comparison, each figure includes moments obtained from the random phase versions of the corresponding simulations. It is worth noting that $M_{4}(q)=1+2 / n$ for data obeying $n$ dimensional Gaussian statistics, and that slow waves correspond to $n=1$ and shear Alfvén waves to $n=2$ in the limit $k_{\perp} \gg k_{z}$.

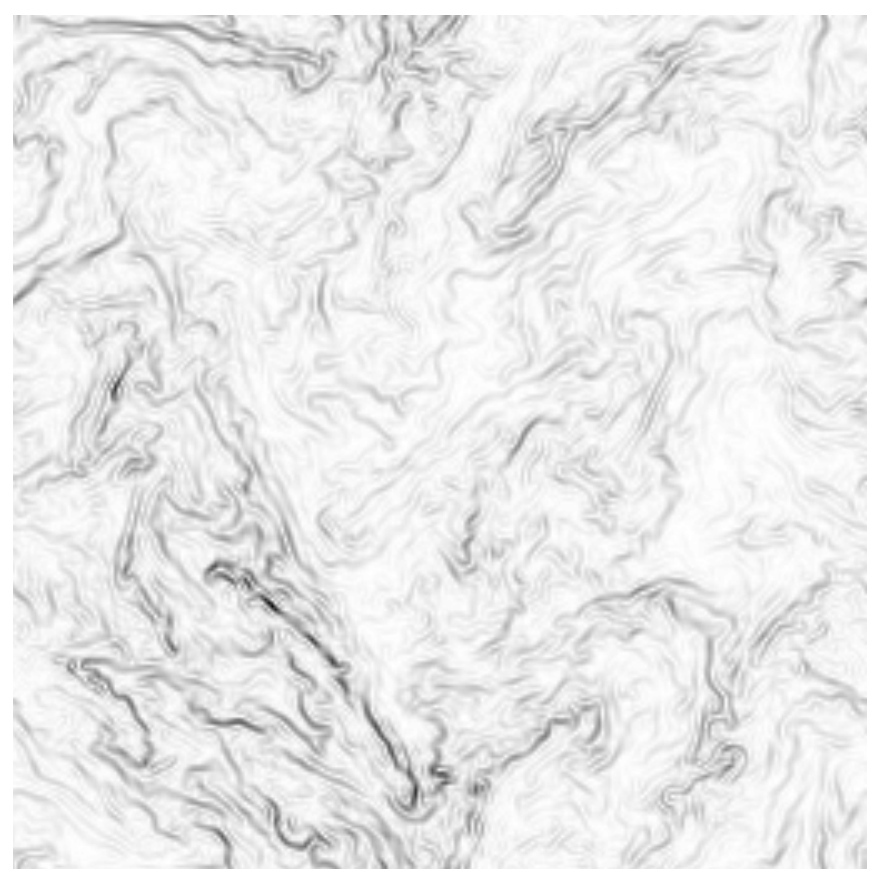

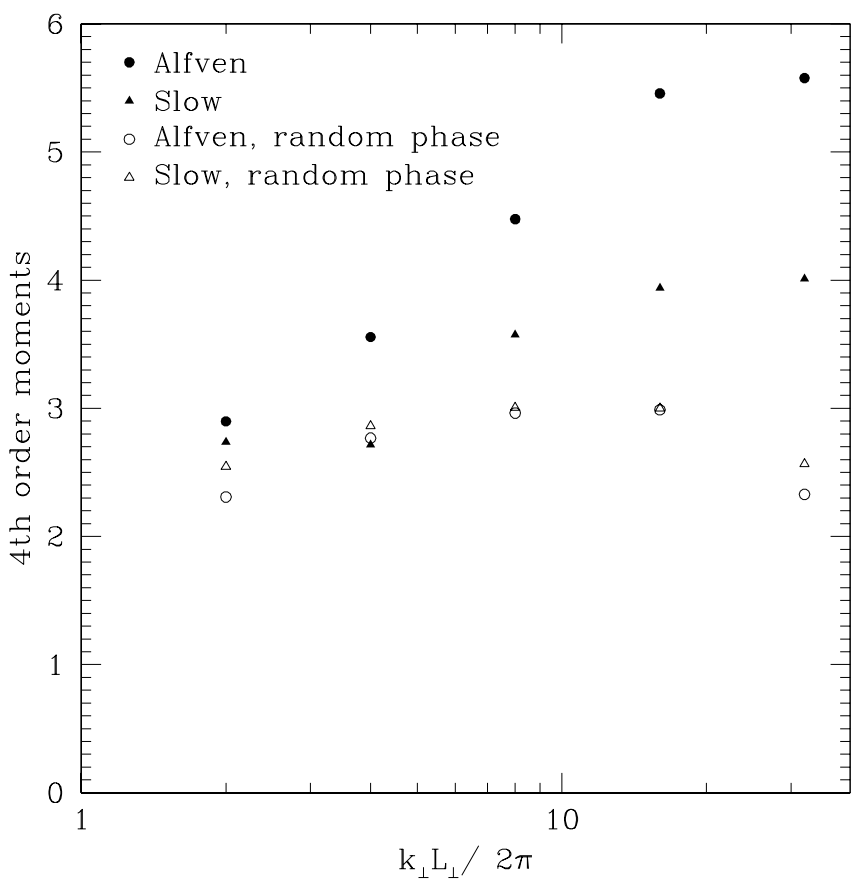

FIG. 23.-Normalized fourth-order moments from gradients of Alfvén and slow modes. Gradients are defined as $\partial_{x} q$, where $q$ is one of the Elsasser fields. Moments obtained from upward- and downwardpropagating waves are averaged. The location of the low-pass filter is denoted by $k_{\perp}$.

\subsubsection{Passive Scalar}

Intermittency also characterizes the concentration of the passive scalar. In the left and right panels of Figure 24, we plot the magnitude of the gradient of the passive scalar computed in our highest resolution simulation F5. The contrast between the simulation and random phase data is striking. Coherent structures that are prominent in the former are absent from the latter.

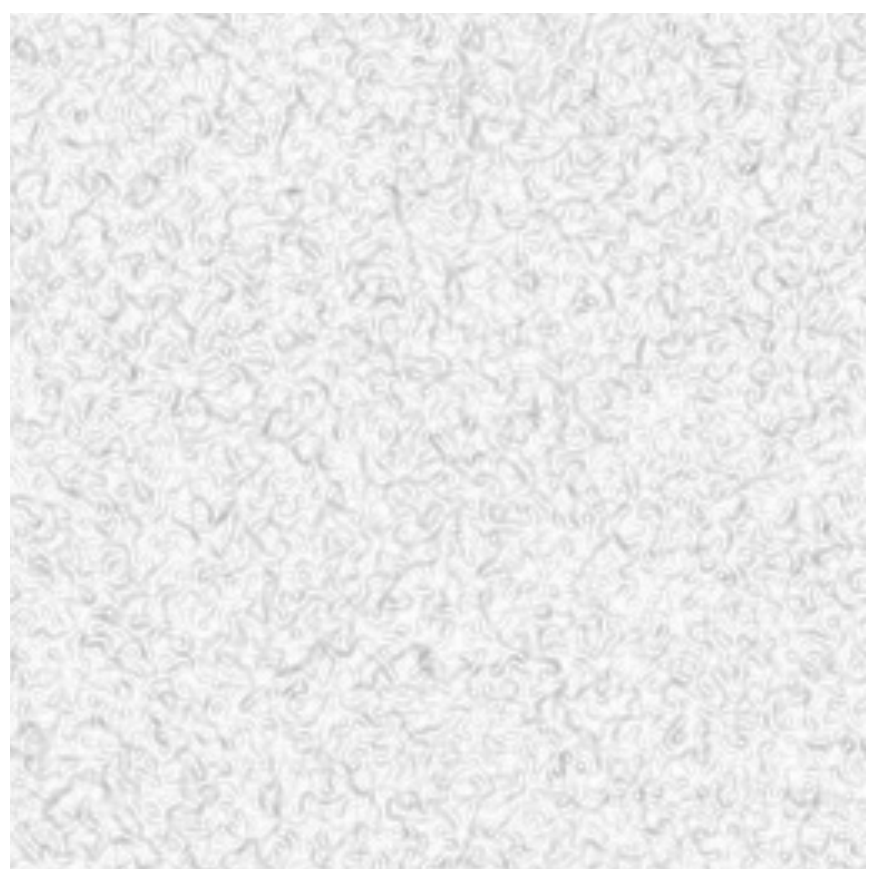

FIG. 24.-Passive scalar gradient magnitude, $|\nabla c|$, from our highest resolution, $256 \times 256 \times 512$, simulation F5. The image plane is $z=0$. Simulation data and their random phase transforms are plotted in the left and right panels, respectively. 


\section{DISCUSSION OF RESULTS}

\subsection{Comparison With GS Model}

The GSI model for the inertial-range cascade of strong MHD turbulence is based on two assumptions:

1. Energy transfer is local in wavenumber space.

2. Linear and nonlinear timescales maintain near equality.

These assumptions lead to two predictions:

1. The one-dimensional energy spectrum $E\left(\boldsymbol{k}_{\perp}\right) \propto k_{\perp}^{-5 / 3}$.

2. The cascade is anisotropic, with energy confined within a cone $k_{\|} \propto k_{\perp}^{2 / 3}$.

Results from simulations presented in $\$ \S 5.1$ and 5.2 agree with some aspects of the GS model and differ with others. An analysis of the reality and meaning of the departures from the GS scalings is presented in $\S 6.6$.

\subsubsection{Power Spectra and Structure Functions}

The one-dimensional power spectra displayed in Figures 3 and 4 exhibit inertial-range slopes, $m_{\mathrm{ps}}$, closer to $-3 / 2$ than to the $-5 / 3$ predicted in GSI. This is consistent with the slopes of the transverse structure functions, $m_{\mathrm{sf}}$, shown in Figure 6 being close to 1/2. Since power spectra and structure functions are related by Fourier transforms, these slopes satisfy $m_{\mathrm{ps}}+m_{\mathrm{sf}}=-1$.

A clear increase of anisotropy with decreasing scale is demonstrated in Figure 7. It is consistent with the prediction by GSI that $\lambda_{\|} \propto \lambda_{\perp}^{2 / 3}$. Cho \& Vishniac (2000) give the initial confirmation of this relation.

\subsubsection{Critical Balance}

Equality of linear and nonlinear timescales, also known as critical balance, predicts that $\lambda_{\|} / v_{\mathrm{A}} \approx \lambda_{\perp} / v_{\lambda_{\perp}}$. Figure 8 shows that the ratio $\chi=\left(\lambda_{\|} v_{\lambda_{\perp}}\right) /\left(\lambda_{\perp} v_{\mathrm{A}}\right)$ maintains a value near unity throughout the inertial range, as predicted in GSI. However, there is a marginal problem of consistency. Together, $\lambda_{\|} \propto \lambda_{\perp}^{2 / 3}$ and $\chi=$ const imply $v_{\lambda_{\perp}} \propto \lambda_{\perp}^{1 / 3}$, but the transverse structure function from which we obtain $v_{\lambda_{\perp}}$ to use in forming $\chi$ yields $v_{\lambda_{\perp}} \propto \lambda_{\perp}^{1 / 4}$.

\subsubsection{Cascade Times}

Two measures of the cascade time are plotted against $k_{\perp}$ in Figure 17; $t_{c} \sim \lambda_{\perp} / v_{\perp}$ and $t_{h} \sim v_{\perp}^{2} / \epsilon$. Each exhibits a power-law dependence on $k_{\perp}$, with the former's slope being steeper than the latter's. For $v_{\perp} \propto \lambda_{\perp}^{1 / 3}$, both $t_{c}$ and $t_{h}$ would be proportional to $\lambda_{\perp}^{-2 / 3}$. However, they are not. Clearly, $v_{\perp} \propto \lambda_{\perp}^{1 / 4}$, which yields $t_{c} \propto \lambda_{\perp}^{3 / 4}$ and $t_{h} \propto \lambda_{\perp}^{1 / 2}$, provides a better, although still imperfect fit. A speculative explanation for the difference between $t_{c}$ and $t_{h}$ is offered in $\S 6.6 .4$.

\subsection{Slow Modes}

\subsubsection{Their Passive Role in Cascade}

The passive role played by slow waves in nearly transverse MHD cascades is neatly illustrated by Figure 12. GSII anticipate this behavior and offer a brief motivation. We provide an intuitive explanation in terms of field-line geometry in $\S 3.3 .2$. A mathematical derivation based on the equations of motion written in terms of Elsasser variables (eq. [19]) is outlined below. Consider the evolution of upward-directed waves in a cascade whose anisotropy is measured by the scale-dependent angle $\Theta \approx k_{\|} / k_{\perp} \ll 1$. The nonlinear terms $\boldsymbol{w}_{\downarrow} \cdot \nabla \boldsymbol{w}_{\uparrow}$ and $\nabla P$ in equation (20) are responsible for their cascade. For comparable magnitudes of slow and shear Alfvén waves, $\boldsymbol{w}_{d} \cdot \nabla \boldsymbol{w}_{u}$ is smaller by a factor of $\Theta$ if $w_{d} \propto s$ than if $w_{d} \propto a$, where $s$ and $a$ are the unit polarization vectors of slow and shear Alfvén waves, as defined in equation (18). Since the $\boldsymbol{w}_{d} \cdot \nabla \boldsymbol{w}_{u}$ term is the sole source of $P$, the same comparison applies to the $\nabla P$ term. Note that these comparisons hold for both shear Alfvén and slow $\boldsymbol{w}_{u}$ waves. Since they are independent of the degree of nonlinearity, they apply to the intermediate MHD cascade as well as to the strong one.

\subsubsection{Lack of Conversion of Shear Alfvén Waves to Slow Waves}

Figure 13 demonstrates that the conversion of shear Alfvén waves to slow waves is of negligible significance in MHD cascades. GSI present the original prediction. A modified version of the argument given there is described below. It compares the rate at which slow waves are created in a balanced cascade composed entirely of shear Alfvén waves to the rate at which the shear Alfvén waves cascade.

Our starting point is the Fourier transformed equation of motion for upward-propagating waves written in terms of Elsasser variables,

$$
\begin{aligned}
& {\left[\frac{\partial}{\partial t}-i \omega(\boldsymbol{k})\right] \tilde{\boldsymbol{w}}_{\uparrow}(\boldsymbol{k})=-\frac{i}{8 \pi^{3}} \int d^{3} k_{1} d^{3} k_{2}\left\langle\tilde{\boldsymbol{w}}_{\uparrow}\left(\boldsymbol{k}_{1}\right)\right.} \\
& \left.\quad-\hat{\boldsymbol{k}}\left[\hat{\boldsymbol{k}} \cdot \tilde{\boldsymbol{w}}_{\uparrow}\left(\boldsymbol{k}_{1}\right)\right]\right\rangle\left[\boldsymbol{k} \cdot \tilde{\boldsymbol{w}}_{\downarrow}\left(\boldsymbol{k}_{2}\right)\right] \times \delta\left(\boldsymbol{k}_{1}+\boldsymbol{k}_{2}-\boldsymbol{k}\right),
\end{aligned}
$$

where $\omega(\boldsymbol{k})=k_{z} v_{\mathrm{A}}$ is the linear frequency of the shear Alfvén and slow waves. The rates of change of the amplitudes of slow and shear Alfvén waves with wavevector $\boldsymbol{k}$ in a cascade of pure shear Alfvén waves are given by

$$
\begin{aligned}
& {\left[\frac{\partial}{\partial t}-i \omega(\boldsymbol{k})\right] \tilde{\boldsymbol{S}}_{\uparrow}(\boldsymbol{k})=-\frac{i}{8 \pi^{3}} \int d^{3} k_{1} d^{3} k_{2}} \\
& \quad \times\left[\hat{\boldsymbol{s}}(\boldsymbol{k}) \cdot \boldsymbol{A}_{\uparrow}\left(\boldsymbol{k}_{1}\right)\right]\left[\boldsymbol{k}_{1} \cdot \boldsymbol{A}_{\downarrow}\left(\boldsymbol{k}_{2}\right)\right] \delta\left(\boldsymbol{k}_{1}+\boldsymbol{k}_{2}-\boldsymbol{k}\right),
\end{aligned}
$$

and

$$
\begin{aligned}
& {\left[\frac{\partial}{\partial t}-i \omega(\boldsymbol{k})\right] \tilde{\boldsymbol{A}}_{\uparrow}(\boldsymbol{k})=-\frac{i}{8 \pi^{3}} \int d^{3} k_{1} d^{3} k_{2}} \\
& \quad \times\left[\hat{\boldsymbol{a}}(\boldsymbol{k}) \cdot \boldsymbol{A}_{\uparrow}\left(\boldsymbol{k}_{1}\right)\right]\left[\boldsymbol{k}_{1} \cdot \tilde{\boldsymbol{A}}_{\downarrow}\left(\boldsymbol{k}_{2}\right)\right] \delta\left(\boldsymbol{k}_{1}+\boldsymbol{k}_{2}-\boldsymbol{k}\right) .
\end{aligned}
$$

Let us compare these two rates. ${ }^{15}$ The magnitude of $\hat{\boldsymbol{s}}(\boldsymbol{k}) \cdot$ $\tilde{\boldsymbol{A}}_{\uparrow}\left(\boldsymbol{k}_{1}\right)$ is smaller than that of $\hat{\boldsymbol{a}}(\boldsymbol{k}) \cdot \tilde{\boldsymbol{w}}_{\uparrow}\left(\boldsymbol{k}_{1}\right)$ by the scaledependent anisotropy factor $\Theta \sim k_{z} / k_{\perp} \ll 1$. Thus, only a fraction $\Theta^{2}\left(k_{\perp}\right) \ll 1$ of the energy in shear Alfvén waves is converted into slow waves as the shear Alfvén waves cascade across $k_{\perp}$. This accounts for the negligible production of slow waves as shown in Figure 13.

\subsection{Dynamics of Imbalance}

The proclivity of MHD cascades for imbalance is a consequence of nonlinear interactions being restricted to collisions between oppositely directed waves.

\footnotetext{
15 The net growth rate of shear Alfvén waves vanishes in a steady state cascade. Restricting the integral to $k_{1} \leq k$ yields the rate at which the amplitude of $\boldsymbol{A}_{\uparrow}$ grows due to the cascading of longer $\left(k_{1}<k\right)$ upwardpropagating shear Alfvén waves.
} 


\subsubsection{Forced Turbulence}

Large fluctuations are observed in the energies of different wave types in simulations of forced turbulence. Nevertheless, the imbalance appears to be bounded. Figure 10 provides an excellent example of this behavior.

A simple dynamical model suffices to capture the essence of imbalance in forced MHD turbulence. It consists of the coupled equations

$$
\frac{d E_{\uparrow}}{d t}=-\frac{E_{\uparrow} E_{\downarrow}^{1 / 2}}{L}+\Lambda \quad \text { and } \quad \frac{d E_{\downarrow}}{d t}=-\frac{E_{\downarrow} E_{\uparrow}^{1 / 2}}{L}+\Lambda,
$$

where $E$ denotes the energy density of the shear Alfvén waves, $L$ the transverse outer scale, and $\Lambda$ the excitation rate. The equilibrium energy density and the nonlinear cascade timescale are defined by $E_{\mathrm{eq}}=(\Lambda L)^{2 / 3}$ and $t_{c}=$ $L^{2 / 3} / \Lambda^{1 / 3}$, respectively.

To investigate the stability of forced balanced cascade, we set

$$
E_{\uparrow}=E_{\mathrm{eq}}+\Delta E_{\uparrow} \quad \text { and } \quad E_{\downarrow}=E_{\mathrm{eq}}+\Delta E_{\downarrow},
$$

and then substitute these expressions into equation (75) to obtain

$$
\begin{gathered}
\frac{d \Delta E_{\uparrow}}{d t}=-\frac{1}{2 t_{c}}\left(2 \Delta E_{\uparrow}+\Delta E_{\downarrow}\right) \quad \text { and } \\
\frac{d \Delta E_{\downarrow}}{d t}=-\frac{1}{2 t_{c}}\left(2 \Delta E_{\downarrow}+\Delta E_{\uparrow}\right) .
\end{gathered}
$$

Assuming a time dependence proportional to $e^{s t}$, we find eigenvalues

$$
s_{1}=-\frac{1}{2 t_{c}} \quad \text { and } \quad s_{2}=-\frac{3}{2 t_{c}} .
$$

This establishes the stability of the forced balanced cascade. It also shows that fluctuations associated with the $s_{1}$ eigenmode decay rather slowly. These characteristics accord well with the runs of Alfvén wave energy densities displayed in Figure 10.

A more sophisticated analysis would include a proper statistical treatment of forcing and an investigation of the spectrum of fluctuations.

\subsubsection{Decaying Turbulence}

Simulations of decaying MHD turbulence exhibit large imbalances. Thus, a perturbation analysis is inappropriate. Fortunately, for $\Lambda=0$ equation (75) admits an analytic solution. As is easy to verify by direct substitution, $\Delta E^{1 / 2} \equiv$ $E_{\uparrow}^{1 / 2}-E_{\downarrow}^{1 / 2}=\Delta E_{0}^{1 / 2}$ is a constant, and

$$
\frac{d}{d t} \ln \left(\frac{E_{\uparrow}}{E_{\downarrow}}\right)=\frac{\Delta E_{0}^{1 / 2}}{L} .
$$

Thus, imbalance grows exponentially in decaying turbulence. This accounts qualitatively for the behavior seen in Figure 11.

Dobrowolny, Mangeney, \& Veltri (1980) propose the growth of imbalance in decaying MHD turbulence as an explanation for the fact that the preponderance of shear
Alfvén waves in the solar wind propagate outward along the interplanetary magnetic field. Support for this proposal is provided by simulations described in Pouguet, Meneguzzi, \& Frisch (1986).

\subsubsection{Axial Asymmetry}

Axial asymmetry refers to a net polarization of shear Alfvén waves. This can occur even in a balanced cascade. MHD cascades have a tendency to develop axial asymmetry because the strength of nonlinear interaction between oppositely directed shear Alfvén waves 1 and 2 is proportional to $\hat{\boldsymbol{a}}\left(\boldsymbol{k}_{1}\right) \cdot \hat{\boldsymbol{a}}\left(\boldsymbol{k}_{2}\right)$ (see eq. [74]). Thus, the interaction vanishes for parallel polarizations and is strongest for orthogonal polarizations.

Decaying turbulence is unstable to the growth of axial asymmetry. Waves with the subdominant polarization cascade more rapidly that those with the dominant polarization. Axial asymmetry is bounded in forced turbulence. However, correlated fluctuations occur across the inertial range within regions of spatial scale comparable to the outer scale, since the cascade tends to preserve polarization alignment.

\subsection{Intermittency}

Tubes of high vorticity, often referred to as worms, are prominent features in simulations of hydrodynamic turbulence. Worms have diameters of the order of the dissipation scale and lengths approaching the outer scale. They are thought to form from the rolling up of vortex sheets. In spite of their prominence, worms do not affect the inertial-range dynamics (Jimenez et al. 1993).

Coherent structures are also evident in MHD simulations. Examples are shown in $\S 5.7$, where the magnitudes of the curls of the dynamical fields and of the gradient of the passive scalar are plotted in $(x, y)$ slices. These regions have narrow dimensions, comparable to the dissipation scale and lengths approaching the outer scale $L_{\perp}$. In these respects they resemble worms. We suspect that these structures are vortex sheets that extend along the $z$-axis and that the magnetic field prevents them from rolling up. Their correlation lengths along $z$ are unresolved. However, this is not a strong constraint, since $k_{M_{z}} L_{\perp} \approx 0.3$.

\subsection{Comparison with Previous Simulations}

Shebalin et al. (1983) report the development of anisotropy in isotropically excited MHD. Their simulations are two-dimensional, with one axis parallel to the direction of the mean magnetic field. Thus, they are composed entirely of slow waves. An isotropic distribution of slow waves will initiate an anisotropic cascade. However, nonlinear interactions among slow waves weaken as the cascade becomes more transverse because their strength is proportional to coefficients such as $\boldsymbol{k}_{2} \cdot \boldsymbol{S}_{\downarrow}\left(\boldsymbol{k}_{1}\right)$, and for $\boldsymbol{k} \rightarrow \boldsymbol{k}_{\perp}, \hat{\boldsymbol{s}} \rightarrow \hat{\boldsymbol{z}}^{16}$ Convincing demonstrations of the development of anisotropy in fully three-dimensional MHD simulations are presented in Oughton, Priest, \& Matthaeus (1994) and Matthaeus et al. (1998). Each of these papers provides evidence that anisotropy increases at smaller scales. Each also claims that up to a saturation limit, anisotropy is more

\footnotetext{
16 This remains true in three dimensions.
} 
pronounced the larger the ratio of mean to fluctuating magnetic field strength. Matthaeus et al. note that this latter trend is inconsistent with the scaling for anisotropy proposed by GSI.

An analysis by Cho \& Vishniac (2000) clears up the confusion regarding the scale dependence of anisotropy in MHD turbulence. Unlike previous workers, who measured anisotropy in coordinate systems fixed to the sides of their computational boxes, Cho \& Vishniac compute anisotropy in local coordinate frames tied to the direction of the total magnetic field. We follow their technique of using structure functions computed in directions parallel and perpendicular to that of the local magnetic field (see $\S$ 5.2.1). Both they and we find results that are consistent with the relation $\lambda_{\|} \propto$ $\lambda_{\perp}^{2 / 3}$ proposed by GSI. However, the ratio of the fluctuating to mean field is $\sim 0.5$ in their simulations and $\sim 0.01$ in ours.

\subsection{Departure from GS Scalings}

The GS scalings lead to the unambiguous prediction of a scale-dependent anisotropy $k_{\|} \propto k_{\perp}^{2 / 3}$, and a onedimensional Kolmogorov spectrum $E\left(k_{\perp}\right) \propto k_{\perp}^{-5 / 3}$. While our simulations are in accord with the former, they consistently indicate that the one-dimensional power spectrum has an index closer to $3 / 2$ than $5 / 3$.

We do not know whether our simulation method is producing an anomalous power law or whether we have discovered a feature of MHD turbulence that is not incorporated in the GS scalings. We first examine several effects that might produce anomalous power laws in numerical simulations. Then we offer some speculations about the role of intermittency.

\subsubsection{Forcing}

Borue \& Orsag (1994) attribute the $k^{-1.85}$ inertial range they find for a simulation of hydrodynamic turbulence to temporal intermittency associated with forcing. We are unable to discern any difference in the inertial-range slopes from our simulations of forced and decaying MHD turbulence. As an example, compare the shear Alfvén wave spectra from both the forced simulation F1 and the decaying simulation D3, which are plotted in Figure 13.

\subsubsection{Dealiasing}

Energy loss in our simulations is due to a combination of dealiasing and hyperviscosity, as illustrated in Figure 9 for $\mathrm{F} 2$, one of our intermediate-resolution simulations. Their relative importance depends on the parameter $\bar{v}(\pi N / 2)^{1 / 3}$, defined in equation (68). With our choice of viscosity parameters, dealiasing contributes relatively less of the energy loss in our lowest resolution simulation F1 and relatively more in our highest resolution simulation F5. However, all of our simulations show similar inertial-range slopes, as a comparison of Figures 3, 4, and 13 verifies. If there is any significant difference, it is that F5, the highest resolution simulation, for which dealiasing is least important, has the steepest inertial-range slope.

\subsubsection{Hyperviscosity}

Hyperviscosity applied in hydrodynamic simulations is known to produce a spurious flattening of the onedimensional inertial-range slope over a range of $k$ approaching the viscous cutoff (Borue \& Orsag 1994). A weaker form of this bottleneck effect is apparent in the simulations of MHD turbulence described by Cho \& Vishniac (2000). Both Borue \& Orsag (1994) and Cho \& Vishniac (2000) employ an eighth-order hyperviscosity. Our simulations, which use a fourth-order hyperviscosity, show no indication of a bottleneck effect. This can be seen from the absence of flattening of the inertial-range slope at high $k_{\perp}$ in Figures 3 and 4.

Could hyperviscosity flatten the slope across the entire inertial range? Muller \& Biskamp (2000) suggest that it does. They present the results of a $512^{3}$ simulation with nearly isotropic forcing that uses first-order (physical) viscosity. It exhibits an inertial-range slope slightly steeper than $5 / 3$. Then they mention that a similar calculation done with second-order hyperviscosity results in a flatter inertial range. To test the effect of hyperviscosity on the inertialrange slope, we carry out simulation F6, which uses physical viscosity but is otherwise similar to simulation F2. The one-dimensional spectrum from this simulation is plotted in Figure 25. Its inertial range appears to have a slope closer to $3 / 2$ than $5 / 3$. This is not entirely conclusive, because the inertial range is truncated at the low- $k_{\perp}$ end by forcing and at the high $-k_{\perp}$ end by viscosity. Higher resolution simulations, which are beyond our current computational resources, are needed to settle this issue.

\subsubsection{Speculations}

For the moment, let us accept the shallow inertial-range slope as a real feature of MHD turbulence. How might it be accounted for? An intriguing possibility is that the nonlinear interactions responsible for the cascade become increasingly intermittent with decreasing scale.

A given degree of spatial intermittency in the energy density is likely to have more serious consequences for the turbulent cascade in MHD than in HD. In HD, energy cascades according to the local value of $\lambda / v$. But in MHD,

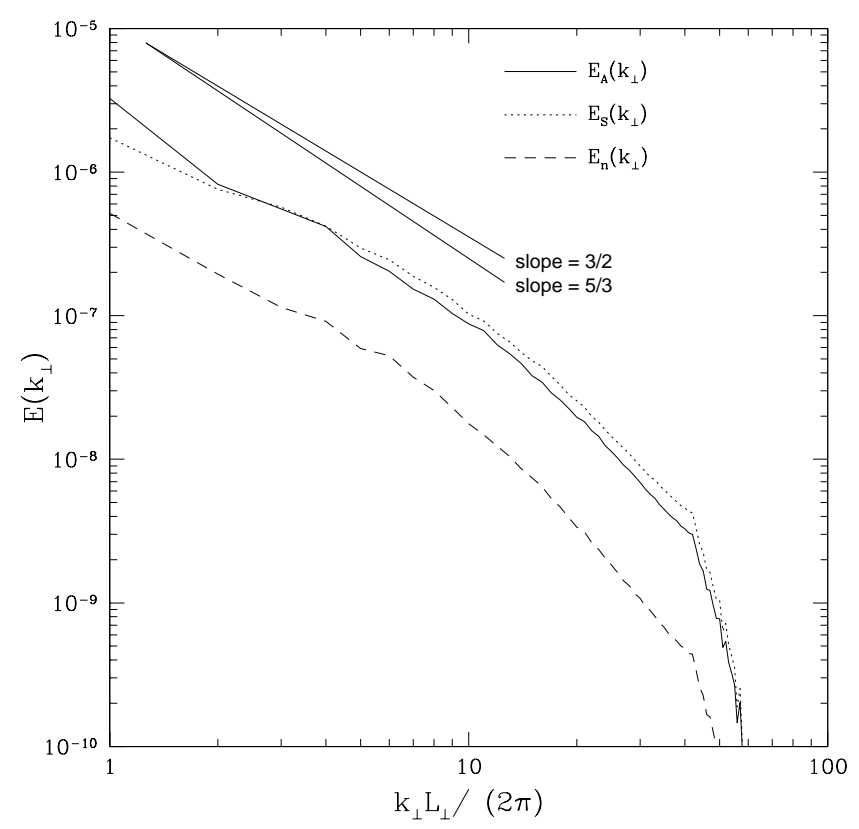

FIG. 25.-Inertial range computed with ordinary viscosity. Simulation F6 with $v_{1}=2 \times 10^{-4}$ and resolution $128 \times 128 \times 512$ is similar to simulation $\mathrm{F} 2$, but uses ordinary viscosity instead of hyperviscosity. 
nonlinear interactions are restricted to collisions between oppositely directed wave packets. Thus, if the spatial filling factor of the energy density, $f$, is small, that of the interactions, $f^{2}$, is smaller still. This may account for the shallower slope of $t_{h} \sim v_{\perp}^{2} / \epsilon$ as compared to $t_{c} \sim \lambda_{\perp} / v_{\perp}$, seen in Figure 17 and discussed in $\S$ 6.1.3.

Research reported in this paper was supported by NSF grant 94-14232 and a NSF Graduate Research Fellowship held by J. M. We thank Ben Chandran, Steve Cowley, Andrei Gruzinov, Reuben Krasnopolsky, Russell Kulsrud, Yoram Lithwick, and Dale Pullin for informative discussions. We owe special thanks to an anonymous referee whose superb review led to a great improvement of the manuscript. Our simulations were carried out on supercomputers operated by the helpful staff at the Caltech Center for Advanced Computing Resources. Edith Huang provided especially valuable advice on their use.

\section{APPENDIX}

\section{A1. SPECTRAL NOTATION}

Cartesian coordinates are distinguished by Greek indices that run from 1 to 3. Simulations are carried out in boxes whose sides have lengths $L_{\alpha}$, which are partitioned by $N_{\alpha}$ grid points. Integer coordinate components, $l_{\alpha}$, and integer wavevector components, $s_{\alpha}$, are defined through the relations

$$
x_{\alpha}=\frac{L_{\alpha}}{N_{\alpha}} l_{\alpha}, \quad \text { where } 0 \leq l_{\alpha}<N_{\alpha},
$$

and

$$
k_{\alpha}=\frac{2 \pi}{L_{\alpha}} s_{\alpha}, \quad \text { where }-\frac{N_{\alpha}}{2} \leq s_{\alpha} \leq \frac{N_{\alpha}}{2} .
$$

The discrete Fourier transform in one dimension is given by

$$
\tilde{q}(s)=\frac{1}{N} \sum_{l} q(l) e^{2 \pi i s l / N},
$$

where the tilde denotes Fourier transform. Generalization to three dimensions is trivial.

\section{A2. SPECTRAL ALGORITHM}

\section{A2.1. Fourier Space Equations}

We evolve the incompressible MHD equations in Fourier space, where they take the form (Lesieur 1990)

$$
\begin{gathered}
\left.\partial_{t} \tilde{v}_{\alpha}=-i k_{\gamma}\left(\delta_{\alpha \beta}-\frac{k_{\alpha} k_{\beta}}{k^{2}}\right) \widetilde{\left(v_{\beta} v_{\gamma}\right.}-\widetilde{b_{\beta} b_{\gamma}}\right)-v_{n} k^{2 n} \tilde{v}_{\alpha}, \\
\partial_{t} \tilde{b}_{\alpha}=-i k_{\beta}\left(\widetilde{v_{\beta} b_{\alpha}}-\widetilde{b_{\beta} v_{\alpha}}\right)-v_{n} k^{2 n} \tilde{b}_{\alpha}, \\
k_{\alpha} \tilde{v}_{\alpha}=0 \\
k_{\alpha} \tilde{b}_{\alpha}=0 \\
\partial_{t} \tilde{c}=-i k_{\beta} \widetilde{v_{\beta} c}-v_{n} k^{2 n} \tilde{c} .
\end{gathered}
$$

\section{A2.2. Integration Method}

Equations (A4), (A5), and (A8) constitute a system of ordinary differential equations with time as the dependent variable and the Fourier coefficients $\left\{\tilde{v}_{\alpha}, \tilde{b}_{\alpha}, \tilde{c}\right\}$ as the independent variables. We employ a modified version of the second-order Runge-Kutta algorithm (RK2) to advance the variables in time. First-order algorithms are substantially less stable than RK2 at the same time step.

RK2 advances the variables across an interval $\Delta t$ in two stages. Derivatives evaluated at the initial time are used to compute trial values of the variables at the midpoint, $\Delta t / 2$. Then derivatives computed at $\Delta t / 2$ with these trial values are used to advance the variables from $t=0$ to $\Delta t$. In symbolic form,

$$
\tilde{q}_{\text {trial }}(\Delta t / 2)=\tilde{q}(0)+\partial_{t} \tilde{q}(0) \Delta t / 2
$$

is followed by

$$
\tilde{q}(\Delta t)=\tilde{q}(0)+\partial_{t} \tilde{q}(\Delta t / 2) \Delta t
$$


where $\partial_{t} \tilde{q}(\Delta t / 2) \Delta t$ is evaluated using $\tilde{q}_{\text {trial }}(\Delta t / 2)$. Each stage involves a first-order Euler (E1) step, in which the derivative is taken to be constant.

We make one departure from standard RK2 and treat diffusive terms with an integrating factor. Consider an equation of the form

$$
\partial_{t} \tilde{q}(k)=A-v_{n} k^{2 n} \tilde{q}(k),
$$

where $A$ comprises the nondiffusive terms. Its solution, with $A$ constant throughout the interval $\Delta t$, is

$$
\tilde{q}(\Delta t)=\left[\tilde{q}(0)+\frac{A}{v_{n} k^{2 n}}\left(e^{v_{n} k^{2 n} \Delta t}-1\right)\right] e^{-v_{n} k^{2 n} \Delta t} .
$$

We use this expression in place of E1 in each stage of RK2. To lowest order in $v_{n} k^{2 n} \Delta t$, equation (A12) reduces to E1. However, it has the advantage that it yields stable solutions to equation (A11) with constant $A$ for arbitrary values of $v_{n} k^{2 n} \Delta t$, whereas E1 yields unstable solutions for $v_{n} k^{2 n} \Delta t>2$.

\section{A2.3. Dealiasing}

Bilinear terms in equations (A4), (A5), and (A8) are calculated by transforming the individual fields to real space, carrying out the appropriate multiplications there, and then transforming the products back to Fourier space. This requires $N_{1} N_{2} N_{3} \log N_{1} \log N_{2} \log N_{3}$ operations using the fast Fourier transform (FFT) algorithm; $\left(N_{1} N_{2} N_{3}\right)^{2}$ operations would be needed to carry out the equivalent convolution in Fourier space.

This economy comes at the price of either a one-third reduction in resolution or an aliasing error (Canuto 1988). To appreciate this, consider the one-dimensional product

$$
\begin{aligned}
\widetilde{p q(s)} & =\frac{1}{N} \sum_{l}\left[\sum_{s^{\prime}} \tilde{p}\left(s^{\prime}\right) e^{-2 \pi i s^{\prime} l / N} \sum_{s^{\prime \prime}} \tilde{q}\left(s^{\prime \prime}\right) e^{-2 \pi i s^{\prime \prime} l / N}\right] e^{2 \pi i s l / N} \\
& =\frac{1}{N} \sum_{s^{\prime}} \sum_{s^{\prime \prime}} \tilde{p}\left(s^{\prime}\right) \tilde{q}\left(s^{\prime \prime}\right) e^{2 \pi i\left(s^{\prime}+s^{\prime \prime}\right) l / N} \delta_{s, s^{\prime}+s^{\prime \prime}+m N},
\end{aligned}
$$

where $m$ is any integer. The $m=0$ terms comprise the convolution, and the remainder the aliasing error. To avoid the aliasing error, we set all Fourier components with $|s|>N / 3$ to zero both before we compute the real-space fields and again after we return the bilinear terms to Fourier space. Truncation ensures that Fourier components of bilinear terms with $m \neq 0$ vanish. Its cost is the reduction of the effective spatial resolution from $N$ to $2 N / 3$.

\section{A3. TESTS OF THE SPECTRAL CODE}

Time derivatives of field quantities computed with the spectral code agree with those obtained from a finite-difference program with an elliptic incompressible pressure operator. Although the latter is unstable, it offers an independent method for computing time derivatives. The code preserves the solinoidal character of $v$ and $\boldsymbol{b}$. To machine accuracy, it returns $\boldsymbol{k} \cdot \partial_{t} \tilde{\boldsymbol{v}}(\boldsymbol{k})=0$ and $\boldsymbol{k} \cdot \partial_{t} \tilde{\boldsymbol{b}}(\boldsymbol{k})=0$. It also conserves energy. Provided that $v_{n}=0$, it yields $\partial_{t} E=\sum_{\boldsymbol{k}} \tilde{\boldsymbol{v}}(\boldsymbol{k}) \cdot \partial_{t} \tilde{\boldsymbol{v}}(\boldsymbol{k})+$ $\tilde{\boldsymbol{b}}(\boldsymbol{k}) \cdot \partial_{t} \tilde{\boldsymbol{b}}(\boldsymbol{k})=0$, again to machine accuracy. Harmonic Alfvén waves are evolved by our spectral code in a manner consistent with their analytic dispersion relation.

Results obtained from a simulation of decaying hydrodynamic turbulence (Z45) run with our code agree with those from a more thorough simulation by Jimenez et al. (1993). Our simulation is carried out in a cubic box with $L=1.0$, has resolution $256^{3}$, kinematic viscosity $v=8 \times 10^{-4}$, time step $\Delta t=2.5 \times 10^{-4}$, and is initialized with rms velocity $v=1.0$. We compute components of the velocity gradient longitudinal, $\nabla_{\|} v_{\|}$, and transverse, $\nabla_{\perp} v_{\|}$, to $v$ at each point in our computational box. Distribution functions, $P F_{q}(x)$, of each quantity, $q$, are compiled and moments calculated according to

These are shown in Table 4.

$$
M_{n}=\frac{\int_{-\infty}^{\infty} x^{n} P F_{q}(x)}{\left[\int_{-\infty}^{\infty} x^{2} P F_{q}(x)\right]^{n / 2}} .
$$

\begin{tabular}{|c|c|c|c|c|c|c|c|}
\hline \multirow[b]{2}{*}{$n$} & \multicolumn{3}{|c|}{ SimULATION Z45 } & \multicolumn{3}{|c|}{ JIMINEZ ET AL. } & \multirow[b]{2}{*}{ GAUSSIAN } \\
\hline & $v$ & $\nabla_{\|} v_{\|}$ & $\nabla_{\perp} v_{\|}$ & $v$ & $\nabla_{\|} v_{\|}$ & $\nabla_{\perp} v_{\|}$ & \\
\hline $3 \ldots \ldots$ & 0 & -0.43 & 0 & 0 & -0.50 & 0 & 0 \\
\hline $4 \ldots \ldots$ & 1.6 & 4.4 & 5.9 & 2.8 & 4.6 & 6.19 & 3 \\
\hline $5 \ldots \ldots$ & 0 & -6.5 & 0 & 0 & -8.0 & 0 & 0 \\
\hline $6 \ldots \ldots$ & 3.4 & 48 & 102 & 13.0 & 55 & 110 & 15 \\
\hline
\end{tabular}

TABLE 4

Tests of Spectral Code 
Because our simulation is of decaying turbulence, whereas that of Jiminez et al. is forced, appropriate comparisons are restricted to inner scale quantities derived from components of $\nabla v$, and exclude outer scale quantities derived from components of $v$. With this proviso, our results are in satisfactory agreement with theirs.

\section{A4. CATAlOG OF SIMUlations}

\section{A4.1. Simulations of Forced Turbulence}

Our basic simulations include forcing at a total power per unit mass of $\mathscr{P}=2 \times 10^{-5}$. Recall that $\rho=1$ and $v_{\mathrm{A}}=1$. Statistically equal power is input into shear Alfvén and slow waves propagating in opposite directions along the magnetic field. Higher resolution simulations run for shorter times are initialized by refining lower resolution simulations run for longer times.

Our sequence of forced simulations begins with $\mathrm{F} 1$, which has resolution $64 \times 64 \times 256$ and runs up to $t=6.6$. Initial condition for simulations $\mathrm{F} 2, \mathrm{~F} 3$, and F4 with resolutions $128 \times 128 \times 512$ are drawn from $\mathrm{F} 1$ at $t=2.4,4.7$, and 6.6, respectively. These are times at which the fluxes of oppositely directed shear Alfvén waves in F1 nearly balance. The refined simulations are run for an additional $\Delta t=0.4$, long enough for small-scale structure to develop up to the dealiasing cutoff. Our highest resolution, $256 \times 256 \times 512$, simulation $\mathrm{F} 5$ is initialized from $\mathrm{F} 2$ at $t=2.8$ and run until $t=2.95$.

\section{A4.2. Simulations of Decaying Turbulence}

Our simulations of decaying turbulence are designed to test specific properties of the MHD cascades. Simulation D1 continues F1 without forcing from $t=2.8$ to 9.9. Simulations D2 and D3 are initialized from F1 at $t=6.6$, the former by removing the Alfvén down and slow up-waves, and the latter by removing all slow waves. A series of simulations are initialized from forced simulations by removing all upward-propagating waves outside a specified band, while leaving the down-modes unchanged. Simulations D4, D5, D6, and D7 are each initialized from F2 at $t=2.8$, with the up-modes band-filtered from $2 \leq s_{\perp} \leq 4,4 \leq s_{\perp} \leq 8,8 \leq s_{\perp} \leq 16$, and $16 \leq s_{\perp} \leq 32$, respectively. Likewise, simulations D8 and D9 are initialized from F5 at $t=2.95$ with up-modes band-filtered from $16 \leq s_{\perp} \leq 32$ and $32 \leq s_{\perp} \leq 64$, respectively. The former has the same up-band as D7 but twice the transverse resolution.

\section{REFERENCES}

Berezinskii, V. S. 1990, Astrophysics of Cosmic Rays (Amsterdam: NorthHolland)

Blandford, R. D., \& Eichler, D. 1987, Phys. Rep., 154, 1

Borue, V., \& Orszag, S. Z. 1995, Europhys. Lett., 29, 687

Canuto, C. 1988, Spectral Methods In Fluid Dynamics (Berlin: Springer)

Chandran, B. D. G. 2000, Phys. Rev. Lett., 85, 4656

Cho, J., \& Vishniac, E. T. 2000, ApJ, 539, 273

Dobrowolny, M., Mangeney, A., \& Veltri, P. 1980, Phys. Rev. Lett., 45, 144

Goldreich, P., \& Sridhar, S. 1995, ApJ, 438, 763 (GSI) . 1997, ApJ, 485, 680 (GSII)

Higdon, J. C. 1984, ApJ, 285, 109

Iroshnikov, P. S. 1963, AZh, 40, 742

Jimenez, J., Wray, A. A., \& Saffman, P. G. 1993, J. Fluid Mech., 255, 65

Kolmogorov, A. N. 1941, Dokl. Akad. Nauk SSSR, 30, 9

Kraichnan, R. H. 1965, Phys. Fluids, 8, 1385
Lazarian, A., \& Vishniac, E. T. 1999, ApJ, 517, 700

Lesieur, M. 1990, Turbulence In Fluids (Dordrecht: Kluwer)

Matthaeus, W. H., Oughton, S., Ghosh, S., \& Hossain, M. 1998, Phys. Rev. Lett., 81, 2056

Müller, W. C., \& Biskamp, D. 2000, Phys. Rev. Lett., 84, 475

Ng, C. S., \& Bhattacharjee, A. 1996, ApJ, 465, 845

Oughton, S., Priest, E. R., \& Matthaeus, W. H. 1994, J. Fluid Mech., 280, 95

Parker, E. N. 1979, Cosmical Magnetic Fields (Oxford: Oxford Univ. Press)

Pouquet, A., Meneguzzi, M., \& Frisch, U. 1986, Phys. Rev. A, 33, 4266

Rickett, B. J. 1990, ARA\&A, 28, 561

Shebalin, J. V., Matthaeus, W. H., \& Montgomery, D. J. J. 1983, J. Plasma Phys., 29, 525

Sridhar, S., \& Goldreich, P. 1994, ApJ, 432, 612 